\title{
Independent gradient model based on Hirshfeld partition (IGMH): A new method for visual study of interactions in chemical systems
}

\author{
Tian Lu, ${ }^{*}$ Qinxue Chen \\ Beijing Kein Research Center for Natural Sciences, Beijing 100022, P. R. China \\ (http://www.keinsci.com) \\ * Correspondence author. E-mail: sobereva@sina.com
}

\section{ORCiD}

Tian Lu: 0000-0002-1822-1229

Qinxue Chen: 0000-0003-0155-2387

\begin{abstract}
The independent gradient model (IGM) originally proposed in Phys. Chem. Chem. Phys., 19, 17928 (2017) has been increasingly popular in visual analysis of intramolecular and intermolecular interactions in recent years, and it has many clear advantages over the widely employed noncovalent interaction (NCI) method, such as intrafragment and interfragment interactions can be elegantly isolated and thus separately studied, the isosurfaces are smoother and less jaggy. However, we frequently observed that there is an evident shortcoming of IGM map in graphically studying weak interactions, that is its isosurfaces are usually too bulgy; in these cases, not only the graphical effect is poor, but also the color on some areas on the isosurfaces is inappropriate and may lead to erroneous analysis conclusions. In addition, the IGM method was originally proposed based on promolecular density, which is quite crude and does not take actual electronic structure into account. In this article, we first present a detailed overview of the IGM analysis, and then propose our new variant of IGM, namely IGM based on Hirshfeld partition of molecular density (IGMH), which replaces the free-state atomic densities involved in the IGM method with the atomic densities derived by Hirshfeld partition of actual molecular electron density. This change makes IGM have more rigorous physical background. In addition, we describe some indices defined on the top of IGM or IGMH framework to quantify contributions from various atoms or atom pairs to interaction between specific fragments. A large number of application examples in this article, including molecular and periodic systems, weak and chemical bond interactions, fully demonstrate the important value of IGMH in intuitively understanding interactions in chemical systems. Comparisons also showed that the IGMH usually has markedly better graphical effect than IGM and overcomes known problems in IGM. Currently IGMH analysis has been efficiently supported in our freely available and user-friendly wavefunction analysis code Multiwfn (http://sobereva.com/multiwfn), and a detailed tutorial is presented. We hope that IGMH will become a new popular method among chemists for exploring interactions in wide variety of chemical systems.
\end{abstract}


Keywords: independent gradient model; non-covalent interaction; weak interaction; reduced density gradient; interaction region indicator; Hirshfeld; Multiwfn 


\section{Introduction}

The non-covalent interaction (NCI) method proposed by Yang and coworkers in 2010 is a very powerful visual analysis technique for weak interactions in chemical systems. ${ }^{[1]}$ It employs isosurfaces of reduced density gradient (RDG) to graphically reveal main occurrence regions of weak interactions, and distinguishes the interaction types by the colors corresponding to the values of mapped function $\operatorname{sign}\left(\lambda_{2}\right) \rho$ on the isosurfaces. Due to the great universality and convenience of the NCI method, it has been extensively employed by chemists in theoretical researches of weak interactions for a wide kinds of systems, such as intramolecular hydrogen-bond, ${ }^{[2,3]}$ intermolecular $\pi-\pi$ stacking, ${ }^{[4]}$ interaction of metal cation with atomic cluster, ${ }^{[5]}$ ligand-protein binding, ${ }^{[6]}$ interactions in molecular crystal, ${ }^{[7]}$ and so on. It is noteworthy that recently, we extended idea of $\mathrm{NCI}$ and proposed interaction region indicator (IRI) method, ${ }^{[8]}$ which is not only able to faithfully reveal weak interactions like NCI, but also able to simultaneously clearly unveil various chemical bond interactions.

In 2017, Hénon et al. proposed the independent gradient model (IGM) method, which has some degree of similarity with NCI. In IGM analysis, interaction regions of different strengths are exhibited by isosurfaces of $\delta g$ function with different values, and $\operatorname{sign}\left(\lambda_{2}\right) \rho$ is also employed as the mapped function to visually distinguish interaction types. In our viewpoint, the key advantage of IGM over NCI and IRI is that intrafragment and interfragment interactions can be revealed individually by $\delta g^{\text {intra }}$ and $\delta g^{\text {inter }}$ functions, respectively, and the fragments involved in the analysis can be defined arbitrarily by researchers according to their practical requirement. This feature makes IGM analysis quite flexible and convenient, since one can focus on studying interactions of their real interest without interference from others. In 2018, the IGM method has been supported by Multiwfn since version $3.5,{ }^{[7]}$ which is a popular wavefunction analysis code developed by us. Another available IGM analysis code is the IGMplot developed by Hénon group. ${ }^{[9]}$ Due to the unique value of IGM analysis and the publicly accessible calculation codes, IGM has attracted increasing attentions from researchers in recent years. So far, more than 160 research articles using Multiwfn to conduct IGM analysis have been published, see Refs. [10-26] for some examples.

During our extensive use of IGM method and according to feedbacks from users of our code, we noticed an evident issue in IGM method, that is, in many cases the isosurfaces of $\delta g$ (or $\delta g^{\text {intra }}$ and $\delta g^{\text {inter }}$ ) function are too bulgy. In these cases not only the graphical effect of IGM map is poor, but also the colors of mapped function $\operatorname{sign}\left(\lambda_{2}\right) \rho$ on the $\delta g$ isosurfaces may lead to erroneous analysis conclusions. This problem will be illustrated later in this article. In addition, the IGM method was initially defined based on the density constructed by superposition of free-state atomic densities, which is often known as promolecular density, or density under promolecular approximation; hence, IGM is in principle unable to reflect actual electronic structure like NCI or IRI, we believe this is another limitation of IGM. We note that Hénon et al. have proposed a variant of IGM defined explicitly based on electronic wavefunction using the so-called gradient-based partitioning ${ }^{[27]}$ however this form of IGM is significantly more complicated than the original one and only limited to single-determinant wavefunction, and the aforementioned issue of $\delta g$ isosurfaces is not eliminated.

In this article, we will describe a new form of IGM recently defined by us, namely IGM based on Hirshfeld partition of molecular density (IGMH). Briefly speaking, IGMH replaces the free-state 
atomic densities involved in the IGM method with atomic densities derived by Hirshfeld partition of actual molecular density. We will illustrate that IGMH displays conspicuously better graphical effect than IGM in many cases, not only the isosurfaces are easier to visually inspect, but also possible misleading conclusion on the interaction type could be fully avoided. Due to the appealing feature of IGMH, this method has already been employed in some researches by Multiwfn users ${ }^{\text {[28- }}$ ${ }^{31]}$ since it was available in Multiwfn 3.7. Moreover, IGMH has a more rigorous physical basis than IGM, because it is defined on the top of actual molecular density rather than promolecular density, the latter one fully neglects electron transfer and polarization during formation of current system from isolated atoms. In addition, in order to raise the analysis to a quantitative level, in this article we will also describe atom $\delta \mathrm{g}$ index, atom pair $\delta \mathrm{g}$ index and their percentage contributions, which are defined based on the framework of IGM or IGMH and will be shown to be particularly useful.

The rest of this article is organized as follows: In Section 2, we first present a clear and systematical overview of the original IGM method, so that readers can then easily understand how IGMH works. After that, we introduce our IGMH method and various related quantitative indices. The implementation and features of IGMH and IGM analyses in Multiwfn will be described in Section 3. In Section 4, we will provide rich application examples of IGMH to illustrate its practical value, and comparison will be made to exhibit advantage of IGMH over IGM and NCI. Finally, we summarize the whole article and make some remarks.

\section{Theory}

\subsection{Overview of IGM method}

To quickly understand basic idea of IGM method, we first look at the simplest homonuclear diatomic molecule, hydrogen molecule. At equilibrium structure, the density distributions of the two atoms in their free-state along the molecular axis are shown in Fig. 1 (a), the sum of the two atomic densities corresponds to promolecular density, whose gradient $(g)$ is exhibited by the black curve in Fig. 1 (b). From Fig. 1 (a) one can clearly find that the gradients of densities of the two atoms in the interatomic bonding region have opposite sign. For example, at the position of $\mathrm{X}=1.2 \AA$, the density gradient of $\mathrm{H} 1$ is negative, while that of $\mathrm{H} 2$ is positive. Therefore, in Fig. 1 (b), the contributions from the two atoms to $g$ largely cancel with each other in the bonding region. Note that at the midpoint of the two hydrogens, $g$ is exactly vanished, this position essentially corresponds to the bond critical point (BCP) in atoms-in-molecules (AIM) theory ${ }^{[32]}$ under promolecular approximation. The $g^{\mathrm{IGM}}$ in Fig. 1(b) denotes IGM type of density gradient, which is defined as the sum of absolute value of density gradient of each atom in their free-states; in other words, sign of gradients is ignored in the sum, and thus the density gradient originating from different atoms will not cancel with each other. Due to this feature, $g^{\mathrm{IGM}}$ is upper limit of $g$, and may be understood as the density gradient of hypothetical non-interacting system. The $\delta g$ function is defined as the difference between $g^{\mathrm{IGM}}$ and $g$, it is plotted as the blue curve in Fig. 1(b). It can be seen that $\delta g$ is relatively large in the interatomic interaction region, and reaches maximum at midpoint of the $\mathrm{H}-\mathrm{H}$ bond. This distribution character of $\delta g$ obviously enables it to be utilized to reveal interaction regions like the RDG function in NCI analysis. For example, Fig. 1(b) shows that the dash line corresponding to $\mathrm{Y}=0.2$ has two intersections with the $\delta g$ curve in the $\mathrm{H}-\mathrm{H}$ bonding region, so, this bonding region could be directly revealed by isosurface of $\delta g=0.2$ a.u. Based on this idea, IGM 
analysis mainly employs isosurfaces of $\delta g$ and its variants to exhibit interactions in a chemical system. Note that it was found that the height of peak of $\delta g$ in an interatomic interaction region has a positive correlation with the interaction strength, ${ }^{[33]}$ therefore $\delta g$ function is also useful in characterizing strength of different interactions.

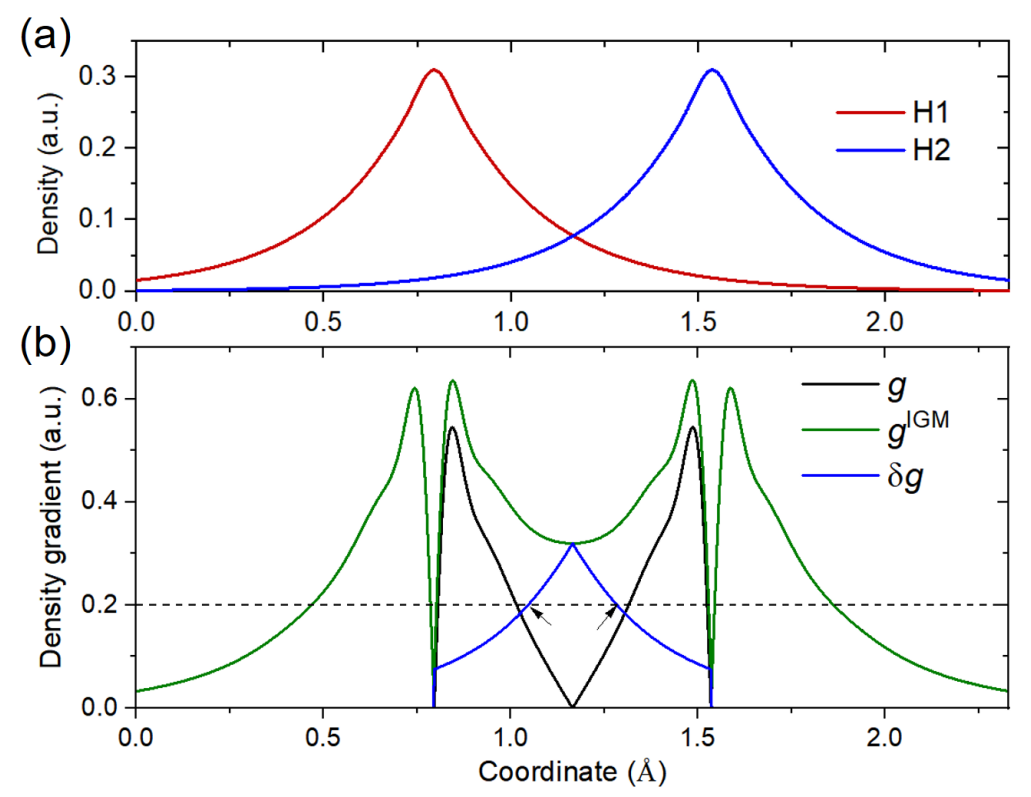

Fig. 1 Density curves along axis of hydrogen molecule at its equilibrium structure. (a) Free-state densities of the two hydrogen atoms (b) Gradient of promolecular density ( $g$ ), IGM type of density gradient $\left(g^{\mathrm{IGM}}\right)$, and their difference $(\delta g)$. The two arrows indicate the intersections between the dash line of $\mathrm{Y}=0.2$ and $\delta g$ curve.

For general, three-dimensional cases, $\delta g$ function can be expressed as

$$
\delta g(\mathbf{r})=g^{\mathrm{IGM}}(\mathbf{r})-g(\mathbf{r})
$$

with

$$
\begin{aligned}
& g(\mathbf{r})=\left|\sum_{i} \nabla \rho_{i}^{\mathrm{free}}(\mathbf{r})\right| \\
& g^{\mathrm{IGM}}(\mathbf{r})=\sum_{i}\left|\nabla \rho_{i}^{\mathrm{free}}(\mathbf{r})\right|
\end{aligned}
$$

where $\mathbf{r}$ denotes Cartesian coordinate vector, $i$ loops over all atoms. The $\rho_{i}^{\text {free }}$ stands for spherically averaged density of atom $i$ in its free state.

The $\delta g$ defined in Eq. (1) reveals all interatomic interactions in a system. The IGM method also defines $\delta g^{\text {inter }}$ focusing on study of interfragment interactions

$$
\delta g^{\text {inter }}(\mathbf{r})=g^{\mathrm{IGM}, \text { inter }}(\mathbf{r})-g^{\text {inter }}(\mathbf{r})
$$

in which $g^{\text {inter }}$ is magnitude of superposition of density gradient of all fragments, while $g^{\mathrm{IGM} \text {,inter }}$ is sum of magnitude of density gradient of all fragments, that is 


$$
\begin{aligned}
& g^{\text {inter }}(\mathbf{r})=\left|\sum_{A} \sum_{i \in A} \nabla \rho_{i}^{\text {free }}(\mathbf{r})\right| \\
& g^{\mathrm{IGM}, \text { inter }}(\mathbf{r})=\sum_{A}\left|\sum_{i \in A} \nabla \rho_{i}^{\mathrm{free}}(\mathbf{r})\right|
\end{aligned}
$$

where $A$ loops over all fragments, and $i$ loops all atoms in the corresponding fragment. It is noteworthy that in the original IGM method only the case of two fragments are considered, while we have generalized the expression of $g^{\text {inter }}$ and $g^{\text {inter,IGM }}$ to the above form so that their difference, $\delta g^{\text {inter }}$, can exhibit interaction between multiple fragments. In IGM analysis the fragments could be arbitrarily defined by researcher according to the character of the system under study and their practical research purpose. Finally, $\delta g^{\text {intra }}$ is defined as complement of $\delta g^{\text {inter }}$ for revealing intramolecular interactions

$$
\delta g^{\text {intra }}(\mathbf{r})=\delta g(\mathbf{r})-\delta g^{\text {inter }}(\mathbf{r})
$$

$\delta g^{\text {inter }}$ is found to be able to reasonably reveal interfragment interactions, ${ }^{[33]}$ the reason is easy to understand from its definition. Its component $g^{\text {inter }}$ takes the sign cancellation effect between the density gradients of the studied fragments into consideration, while the another component $g^{\mathrm{IGM}, \text { inter }}$ fully ignores the cancellation, obviously their difference should be able to reveal interactions between the fragments in their overlap regions. If interaction between two fragments is negligible, the $\delta g^{\text {inter }}$ should be almost vanished everywhere. Since the distribution of $\delta g$ exhibits all interatomic interactions in the present system, if $\delta g^{\text {inter }}$ is subtracted from $\delta g$, the remaining part, namely $\delta g^{\text {intra, }}$, is certainly able to reveal intrafragment interactions.

In IGM analysis, isosurfaces of $\delta g^{\text {inter }}$ and $\delta g^{\text {intra }}$ are commonly used for visualization of interaction regions, and meantime $\operatorname{sign}\left(\lambda_{2}\right) \rho$ function is usually mapped on the isosurfaces by different colors to visually characterize the nature of the interactions. $\operatorname{sign}\left(\lambda_{2}\right) \rho$ denotes product of electron density, $\rho$, and sign of the second largest eigenvalue of Hessian matrix of $\rho, \operatorname{sign}\left(\lambda_{2}\right) . \rho$ in interaction region is generally positively correlated to interaction strength, while $\operatorname{sign}\left(\lambda_{2}\right)$ has certain capacity of distinguishing whether the interaction is attractive or repulsive. ${ }^{[1,7]}$ The common interpretation of $\operatorname{sign}\left(\lambda_{2}\right) \rho$ in different value ranges is given in Fig. 2, and the coloring method in this figure will be employed for all subsequent discussions and examples in this article. Note that the $\lambda_{2}$ and $\rho$ in the original IGM method are evaluated under promolecular approximation.

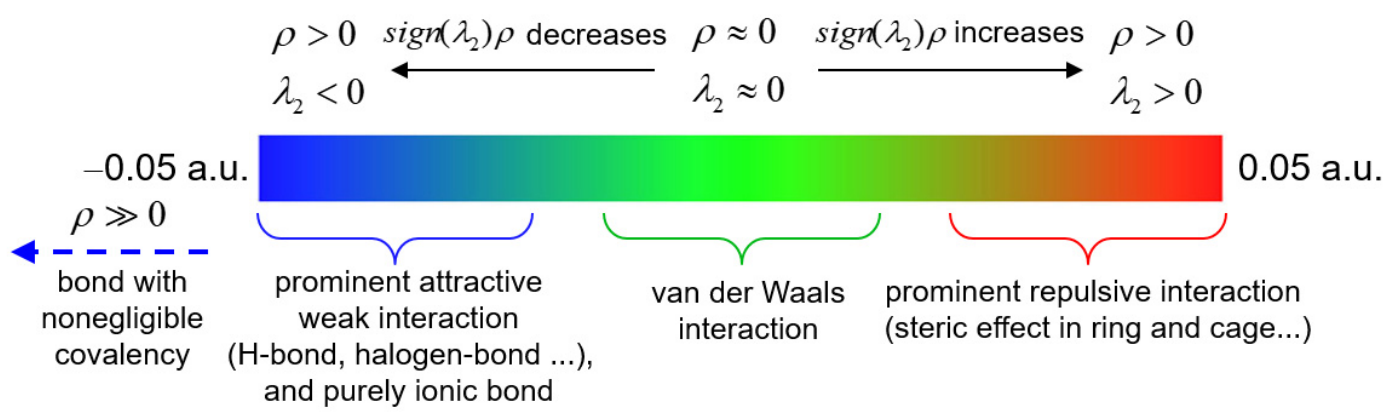

Fig. 2 Common interpretation of coloring method of mapped function $\operatorname{sign}\left(\lambda_{2}\right) \rho$ in IGM and IGMH maps. 


\subsection{Definition of IGMH method}

The original version of IGM was defined purely based on densities of atoms in their free states, namely it is a method under promolecular approximation. We considered whether this method can be defined based on the actual electron density, so that it has a more rigorous physical meaning and can faithfully reflect electronic structure of the system under study. It is noted that Hirshfeld partition is a commonly employed method with a clear physical picture to obtain atomic densities in a chemical system, ${ }^{[34-37]}$ the density of atom $i$ derived in this way is expressed as $\rho_{i}^{\text {Hirsh }}(\mathbf{r})=$ $\rho(\mathbf{r}) w_{i}(\mathbf{r})$, where $\rho$ is actual electron density calculated by quantum chemistry method or determined by crystal diffraction experiments, and the Hirshfeld weighting function of the atom $i$ is defined as

$$
w_{i}(\mathbf{r})=\frac{\rho_{i}^{\mathrm{free}}(\mathbf{r})}{\rho^{\mathrm{pro}}(\mathbf{r})}=\frac{\rho_{i}^{\mathrm{free}}(\mathbf{r})}{\sum_{j} \rho_{j}^{\mathrm{free}}(\mathbf{r})}
$$

where index $j$ loops over all atoms, the $\rho^{\text {pro }}$ in denominator is just the aforementioned promolecular density. The IGMH method proposed in this article differs from the original IGM by two aspects: (1) The free-state atomic density $\rho_{i}^{\text {free }}$ in the IGM formulae is replaced with $\rho_{i}^{\text {Hirsh }}$ (2) The $\operatorname{sign}\left(\lambda_{2}\right) \rho$ is evaluated based on actual density like NCI and IRI methods. Since the IGMH is defined fully based on actual density and gets rid of promolecular approximation, it can be expected that IGMH should have certain practical benefits in exhibiting interactions compared to IGM; this is indeed true, as will be observed in the comparisons given later.

As shown in Eqs. 2 and 4, gradient of free-state atomic density $\left(\nabla \rho_{i}^{\text {free }}\right)$ is involved in IGM analysis, similarly, in the IGMH analysis, gradient of Hirshfeld atomic density $\left(\nabla \rho_{i}^{\text {Hirsh }}\right)$ is needed to evaluate, we found it is easy to implement. Derivative of $\rho_{i}^{\text {Hirsh }}$ with respect to a Cartesian coordinate component $\mu(\mu=x, y, z)$ is

$$
\frac{\partial \rho_{i}^{\text {Hirsh }}}{\partial \mu}=\frac{\partial\left(\rho w_{i}\right)}{\partial \mu}=w_{i} \frac{\partial \rho}{\partial \mu}+\rho \frac{\partial w_{i}}{\partial \mu}
$$

in which the derivative of Hirshfeld weighting function with respect to $\mu$ can be derived as

$$
\begin{aligned}
& \frac{\partial w_{i}}{\partial \mu}=\frac{\partial}{\partial \mu}\left(\frac{\rho_{i}^{\mathrm{free}}}{\sum_{j} \rho_{j}^{\mathrm{free}}}\right)=\frac{\partial \rho_{i}^{\mathrm{free}}}{\partial \mu} \frac{1}{\sum_{j} \rho_{j}^{\mathrm{free}}}-\frac{\rho_{i}^{\mathrm{free}}}{\left(\sum_{j} \rho_{j}^{\mathrm{free}}\right)^{2}} \sum_{j} \frac{\partial \rho_{j}^{\mathrm{free}}}{\partial \mu} \\
& =\frac{1}{\rho^{\mathrm{pro}}} \frac{\partial \rho_{i}^{\mathrm{free}}}{\partial \mu}-\frac{\rho_{i}^{\mathrm{free}}}{\left(\rho^{\mathrm{pro}}\right)^{2}} \sum_{j} \frac{\partial \rho_{j}^{\mathrm{free}}}{\partial \mu}
\end{aligned}
$$

By substituting Eqs. 6 and 8 into Eq. 7, the working equation for Cartesian derivative of $\rho_{i}^{\text {Hirsh }}$ is reached

$$
\frac{\partial \rho_{i}^{\mathrm{Hirsh}}}{\partial \mu}=\frac{\rho_{i}^{\mathrm{free}}}{\rho^{\mathrm{pro}}} \frac{\partial \rho}{\partial \mu}+\frac{\rho}{\rho^{\mathrm{pro}}} \frac{\partial \rho_{i}^{\mathrm{free}}}{\partial \mu}-\frac{\rho \rho_{i}^{\mathrm{free}}}{\left(\rho^{\mathrm{pro}}\right)^{2}} \sum_{j} \frac{\partial \rho_{j}^{\mathrm{free}}}{\partial \mu}
$$

From Eq. 9 it can be seen that compared to IGM, implementation of the $\delta g, \delta g^{\text {inter }}$ and $\delta g^{\text {intra }}$ under the framework IGMH only additionally requires actual density and its first derivative, which are trivial to evaluate when electronic wavefunction is available. 


\subsection{Quantitative indices in the framework of IGM and IGMH}

Given that value of $\delta g$ in an interaction region is closely related to the interaction strength, during our implementation of IGM method in Multiwfn code in 2018, we also designed and implemented atomic pair $\delta g$ index $\left(\delta G^{\text {pair }}\right)$ and atomic $\delta g$ index $\left(\delta G^{\text {atom }}\right)$ under the framework of IGM for quantitatively analyzing interactions. Our tests showed that these indices are also directly compatible with IGMH. We noted that recently Hénon et al. employed similar idea to decompose interactions in host-guest complex into atomic contributions. ${ }^{[38]}$

$\delta G^{\text {pair }}$ is used to quantify the contribution of an atom pair to interaction between two specific fragments. For example, this index between atoms $i$ and $j$, which belong to fragments $A$ and $B$ respectively, is expressed as

$$
\delta G_{i, j}^{\text {pair }}=\int \delta g_{i, j}(\mathbf{r}) \mathrm{d} \mathbf{r}=\int\left[g_{i, j}^{\mathrm{IGM}}(\mathbf{r})-g_{i, j}(\mathbf{r})\right] \mathrm{d} \mathbf{r} \quad i \in A, j \in B
$$

where the integration is carried out over the whole space, and

$$
\begin{aligned}
& g_{i, j}(\mathbf{r})=\left|\nabla \rho_{i}(\mathbf{r})+\nabla \rho_{j}(\mathbf{r})\right| \\
& g_{i, j}^{\mathrm{IGM}}(\mathbf{r})=\left|\nabla \rho_{i}(\mathbf{r})\right|+\left|\nabla \rho_{j}(\mathbf{r})\right|
\end{aligned}
$$

Specifically, in IGM and IGMH analyses, the $\rho_{i}$ in above formulae corresponds to $\rho_{i}^{\text {free }}$ and $\rho_{i}^{\text {Hirsh }}$, respectively.

It is also useful to define percentage atomic pair contribution to interfragment interaction as

$$
\delta G_{i, j}^{\mathrm{pair}}(\%)=\frac{\delta G_{i, j}^{\mathrm{pair}}}{\sum_{k \in A} \sum_{l \in B} \delta G_{k, l}^{\mathrm{pair}}} \times 100 \%
$$

It is noteworthy that commonly the sum of $\delta G^{\text {pair }}$ of all atom pairs between two fragments is not directly equal to the integral of the $\delta g^{\text {inter }}$ between the two fragments, $\delta G^{\text {inter }}$, that is

$$
\sum_{i \in A} \sum_{j \in B} \delta G_{i, j}^{\text {pair }} \neq \delta G_{A, B}^{\text {inter }}=\int \delta g_{A, B}^{\text {inter }}(\mathbf{r}) \mathrm{d} \mathbf{r}
$$

Anyway, as will be illustrated later, there is still practical significance to employ $\delta G^{\text {pair }}$ or $\delta G^{\text {pair }}(\%)$ to highlight "hot" atomic pairs which may have large actual contribution to interaction between the fragments of interest.

For analogous systems, it is expected that there should be a certain degree of positive correlation between the $\delta G^{\text {inter }}$ in the above formula and the magnitude of interaction energy between the considered fragments. So, $\delta G^{\text {inter }}$ could be viewed as a descriptor of interfragment interaction, and may be useful in designing intermolecular interaction potential via machine learning strategy, but this possibility will not be explored in this work.

$\delta G^{\text {atom }}$ is defined to quantitatively measure importance of an atom to interfragment interaction. For example, contribution of atom $i$ in fragment $A$ to interaction between fragments $A$ and $B$ is

$$
\delta G_{i}^{\text {atom }}=\sum_{j \in B} \delta G_{i, j}^{\text {pair }}
$$

the percentage atomic contribution could be defined as

$$
\delta G_{i}^{\text {atom }}(\%)=\frac{\delta G_{i}^{\text {atom }}}{\sum_{j \in A} \delta G_{j}^{\text {atom }}} \times 100 \%
$$

Clearly, when plotting molecule structures, if atoms are colored according to $\delta G^{\text {atom }}$ or $\delta G^{\text {atom }}(\%)$, the relative importance between various atoms to interfragment interactions will be vividly 
displayed. It should be stressed that because the form of the $\delta G^{\text {pair }}$ index is quite simple, positive connection between this index and real interaction energy between atoms cannot always be expected, and hence $\delta G^{\text {atom }}$ index can at most be used to quickly and intuitively exhibit which atoms may play a crucial role for interfragment interaction. To characterize atomic or atomic pair contributions to interfragment interaction in terms of energy, one may consider using energy decomposition analysis based on forcefield (EDA-FF $)^{[26]}$ in Multiwfn code or atomic symmetry adapted perturbation theory $(\mathrm{A}-\mathrm{SAPT})^{[39]}$ in PSI4 code. ${ }^{[40]}$

Recently intrinsic bond strength index (IBSI) was introduced by Hénon et al. based on the IGM of gradient-based partitioning form to characterize strength of chemical bonds, we note this index is also fully compatible with IGMH, but discussion of this index is beyond the scope of the present work.

\section{IGM and IGMH analyses in Multiwfn code}

We have implemented IGM and IGMH analyses in Multiwfn, which is a popular, easy-to-use and freely available wavefunction analysis code. Its precompiled executable files, source code and very detailed manual can be obtained at http://sobereva.com/multiwfn. A detailed and easy-to-learn tutorial of performing these analyses in Multiwfn can be downloaded at http://sobereva.com/multiwfn/res/IGMH tutorial.zip. Various aspects of the IGM and IGMH modules of Multiwfn are described below.

\section{Functions}

Multiwfn possesses the following functions about IGM and IGMH analyses:

- Evenly distributed grid data of $\delta g, \delta g^{\text {inter }}, \delta g^{\text {inter }}$ and $\operatorname{sign}\left(\lambda_{2}\right) \rho$ can be calculated and exported. Their isosurfaces can be directly visualized in Multiwfn, and scripts of VMD visualization program $^{[41]}$ are provided to easily plot $\operatorname{sign}\left(\lambda_{2}\right) \rho$ mapped $\delta g, \delta g^{\text {inter }}$ and $\delta g^{\text {inter }}$ isosurface maps.

- Scatter map between various forms of $\delta g^{\text {inter }}\left(\delta g^{\text {inter }}\right)$ and $\operatorname{sign}\left(\lambda_{2}\right) \rho$ can be directly drawn in Multiwfn, the peaks in this kind of map are shown to be useful in analyzing interfragment (intrafragment) interactions. ${ }^{[33]}$

- $\delta G^{\text {pair }}, \delta G^{\text {pair }}(\%), \delta G^{\text {atom }}, \delta G^{\text {atom }}(\%), \delta G^{\text {inter }}$ indices and IBSI can be estimated. Becke's multicenter numerical integration algorithm ${ }^{[42]}$ is employed to evaluate the integrals in these indices, the numerical accuracy is found to be fairly satisfactory even using inexpensive integration grids (40 radial points and 170 angular points per atom). After calculation, "atmdg.pqr" and "atmdg\%.pqr" files whose "charge" field respectively records $\delta G^{\text {atom }}$ and $\delta G^{\text {atom }}(\%)$ can be exported, so that atoms can then be colored by these indices in VMD program for visual examination of relative importance of various atoms.

- Curve map along a given line, and various types of two-dimensional map (color-filled map, contour map, relief map, etc.) in a given plane, can be directly plotted in Multiwfn for $\delta g$ and $\delta g^{\text {inter }}$.

In addition, due to the rich functions, flexible design and interactive interface of Multiwfn, realizing other kind of analyses relating to IGM and IGMH is possible and may be useful for special research purposes. For example, $\delta g$ value at BCP located by topology analysis module of Multiwfn can be calculated, curve map of $\delta g$ along a specific bond axis or bond path can be plotted and then maximal value on the curve can be directly determined, $\delta g$ and $\delta g^{\text {inter }}$ can be integrated in AIM or electron localization function (ELF) ${ }^{[43,44]}$ basins in basin analysis module, more than one hundred 
of real space functions that supported in Multiwfn can be integrated within isosurface of $\delta g^{\text {inter }}$ via domain analysis module. Therefore, many other IGM or IGMH interaction descriptors that may be valuable in characterizing interactions can be realized by Multiwfn even without modifying source code. Limited to the length of this article, temporarily the value of these descriptors will not be explored in the present work.

\section{Features}

IGM and IGMH analyses in Multiwfn have the following features:

- Definition of fragments is quite flexible. Arbitrary number of fragments is supported and can be defined via a convenient interface, and the union of the fragments is not necessarily equal to the whole system.

- Not only isolated systems but also periodic systems are fully supported in IGM and IGMH analyses, allowing them to investigate any kind of systems, including molecules, materials, interfaces, and so on.

- Since free-state atomic densities for elements from H to $\operatorname{Lr}$ (element 103) have been embedded into Multiwfn, IGM and IGMH analyses can be applied for almost any system. Technically, the spherically averaged atomic radial densities are tabulated in Multiwfn, Lagrangian interpolation is employed to derive density value and its analytical first and second derivatives at given points. High-quality basis sets were employed for generating the atomic densities, and scalar relativistic effect has been taken into account for elements starting from the fourth row via DKH2 Hamiltonian. ${ }^{[45]}$

- The codes relevant to IGM and IGMH analyses have been sufficiently optimized and parallelized by OpenMP technique, and some truncation thresholds of numerical evaluations have been considered for significantly reducing computational cost of large systems without sacrificing accuracy, therefore all aforementioned functions and indices can be evaluated fairly efficiently in Multiwfn.

- The use is extremely simple, users only need to input commands according to the prompts shown on the screen, while writing input files and memorizing keywords are fully avoided. Moreover, the program provides a very user-friendly interface for setting up grid points used for calculating various functions involved in the IGM and IGMH analyses.

It should be emphasized that since the calculation of IGMH relies on electronic wavefunction to evaluate actual density, while IGM is only dependent of the built-in free-state atomic densities, the calculation cost of IGMH is notably higher than that of IGM. But even so, using a common personal computer, there is no any difficulty to perform IGMH analysis in Multiwfn for an organic system consisting of hundreds of atoms. As for IGM analysis, in Multiwfn it can even be applied to huge systems containing as many as hundreds of atoms, such as biomacromolecules.

\section{Input file}

Multiwfn accepts wide variety of file formats as input file of IGM and IGMH analyses:

(1) IGM analysis for isolated systems: Any file format containing atomic coordinates can be used, such as xyz, mol, mol2, pdb, input/output file of Gaussian and ORCA programs, and so on.

(2) IGM analysis for periodic systems: The input file should contain both atomic coordinates and cell information. For example, cif, cub, gro file of GROMACS program, restart file of CP2K program, POSCAR file of VASP program, Gaussian input file containing cell vectors.

(3) IGMH analysis for isolated systems: Any file format containing wavefunction information can be used, such as mwfn, ${ }^{[46]} \mathrm{wfn}$, wfx, fch, molden, GAMESS/Firefly output file, and so on. Most 
popular quantum chemistry programs can export at least one of them, and the exporting method has been carefully documented in Multiwfn manual.

(4) IGMH analysis for periodic systems: The input file should contain both periodic wavefunction and cell information. Molden file exported by CP2K first-principle program ${ }^{[47]}$ after density functional theory calculation is usually used in this context, cell vectors should be manually filled into it as [Cell] field, see Multiwfn manual for details. $\mathrm{mwfn}^{[46]}$ and fch files containing cell vectors are also supported for this purpose.

In principle, IGMH analysis in Multiwfn can be realized at any theoretical level, as long as the inputted wavefunction file carries molecular orbitals or natural orbitals of corresponding level. We found IGMH analysis has a low sensitivity to wavefunction quality. Usually, optimizing the system and producing wavefunction using the popular B3LYP-D3(BJ) ${ }^{[48,49]}$ or $\omega \mathrm{B}^{2} 7 \mathrm{XD}^{[50]}$ exchangecorrelation functional in combination with a medium-sized basis set is fully adequate for IGMH analysis. It is noteworthy that GFN-xTB is a recently popular method at semi-empirical level, which is able to rapidly optimize a system containing hundreds of atoms. ${ }^{[51]}$ The .molden file exported by xtb code ${ }^{[52]}$ using GFN-xTB theory is also supported for IGMH analysis, and the quality of the result based on this very cheap wavefunction is basically acceptable for most cases according to our preliminary tests. IGMH may also be used for revealing interactions for excited states, the requirement on the input file is that it records natural orbitals of the state of interest, which can be easily generated by many popular quantum chemistry programs at time-dependent density functional theory (TDDFT) level, such as Gaussian (via $T D$ and out $=w f n$ keywords). ${ }^{[53]}$

\section{Application examples and comparisons}

Below we will present many application examples for wide variety of systems to illustrate usefulness of IGMH analysis as well as its difference with respect to IGM, NCI and IRI. Unless otherwise specified, for isolated systems, the popular $\omega \mathrm{B} 97 \mathrm{XD}$ exchange-correlation functional ${ }^{[50]}$ in combination with a medium-sized $6-311 \mathrm{G}^{*}$ basis se ${ }^{[54]}$ was used for geometry optimization and generation of the wavefunction for IGMH analysis, Gaussian $16 \mathrm{~A} .03$ program ${ }^{[53]}$ was used for all the density functional theory (DFT) calculations. For periodic systems, CP2K $8.1^{[47]}$ was employed for the DFT calculations using PBE-D3(BJ) exchange-correlation functional ${ }^{[49,55]}$ with $6-311 \mathrm{G}^{* *}$ basis set. ${ }^{[54]}$ All isosurface maps were rendered by VMD 1.9.3 program ${ }^{[41]}$ based on the cube files generated by Multiwfn. Grid spacing of 0.15 Bohr was employed in calculating the grid data involved in IGM and IGMH analyses. In NCI analysis, according to common custom, only the RDG isosurfaces in the regions where electron density is greater than 0.05 a.u. were retained so that only weak interactions are revealed.

\subsection{Revealing weak interactions in molecular systems}

In this section, we focus on using IGMH method to reveal weak interactions in molecular systems. Note that since the strength of these interactions is significantly weaker than that of chemical bonds, a small isovalue of $\delta g^{\text {inter }}$ between 0.003 and 0.01 a.u. is usually suitable for graphically exhibiting the interfragment interaction regions, and it should be adjusted properly to obtain the best graphical effect.

Dodecaphenyltetracene ${ }^{[56]}$ is a recently synthesized molecule with unusual structure character, 
which has a tetracene core encapsulated by as many as twelve peripheral phenyl substituents. The strong steric forces between the spatially crowded phenyl groups make the tetracene highly twisted. We carried out IGM and IGMH analyses for this system and plotted $\operatorname{sign}\left(\lambda_{2}\right) \rho$ colored $\delta g^{\text {inter }}$ isosurface maps, see Fig. 3. The coloring method shown in Fig. 2 is used for this and all IGM/IGMH maps given later. In the calculation, each phenyl group was defined as an individual fragment, therefore in Fig. 3 only the interaction between the twelve phenyl groups are exhibited, which is of our interest. To maximally reveal the interactions between the closely packed aromatic rings in this molecule, isovalue of $\delta g^{\text {inter }}$ is set to 0.005 a.u. in the maps. From the IGMH map, it can be seen that a thin and broad isosurface appears between each pair of adjacent phenyl groups, which very ideally exhibits the $\pi-\pi$ stacking interaction. Although the IGM map in Fig. 3 also displays this character to some extent, there are evident defects. As indicated by the pink arrows, some areas on the isosurfaces show deep blue color, as if there are strong attractive interactions, which in fact are artificial, since it is well known that the $\pi-\pi$ stacking interaction between two six-membered aromatic rings is fairly weak. ${ }^{[57]}$ Obviously, this problem in the IGM map may bring great trouble to researchers in understanding the true interaction nature. The main reason of this issue is that the $\delta g^{\text {inter }}$ isosurfaces of IGM are too bulgy, some parts of the isosurfaces have reached the regions very close to nuclei, where electron density is significantly larger than interatomic interaction regions. This problem of IGM will become more serious when isovalue is set to a smaller value, as in this case the isosurfaces will cover wider spaces. For example, Fig. S1 presents IGM and IGMH maps corresponding to $\delta g^{\text {inter }}$ of 0.003 a.u., the misleading blue areas in the IGM isosurface map become wider than Fig. 3; in contrast, IGMH isosurface map is still fully reasonable even at such a low isovalue, and in this case, the slight steric hindrances between some carbon atoms connected to the tetracene core are also correctly revealed by the red area on the IGMH isosurfaces. It is worth noting that the aforementioned problem of IGM is not caused by the promolecular approximation in $\operatorname{sign}\left(\lambda_{2}\right) \rho$, because we found the problem still exists even if the $\operatorname{sign}\left(\lambda_{2}\right) \rho$ based on actual density is mapped on the IGM $\delta g^{\text {inter }}$ isosurfaces.

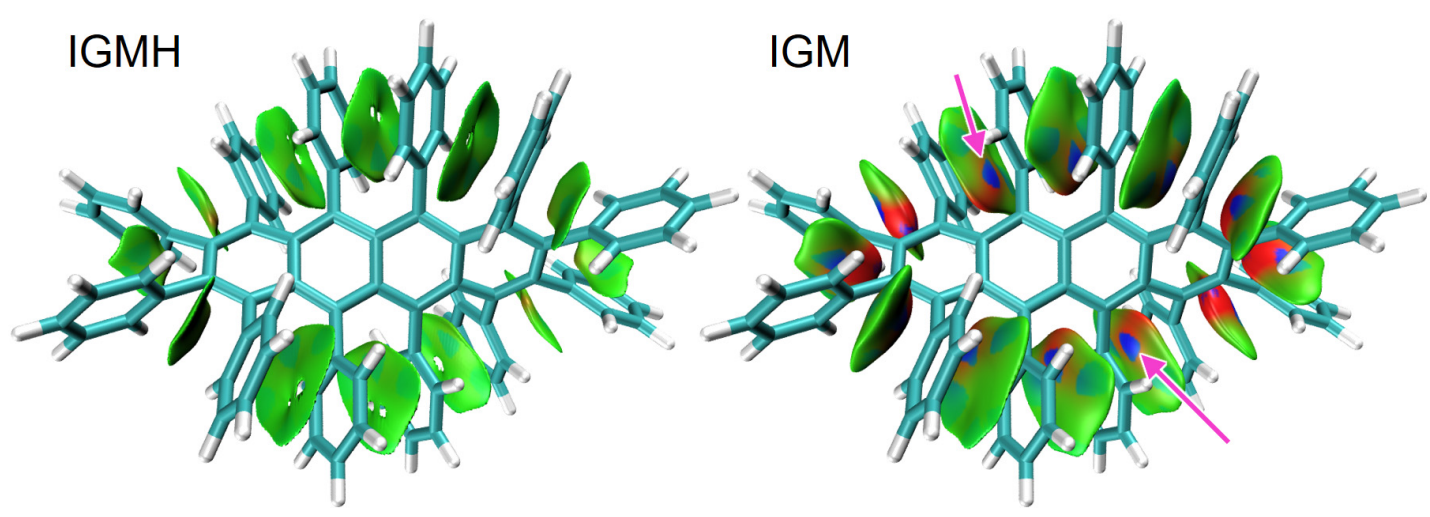

Fig. $3 \operatorname{sign}\left(\lambda_{2}\right) \rho$ colored isosurfaces of $\delta g^{\text {inter }}=0.005$ a.u. of dodecaphenyltetracene corresponding to IGMH and IGM analyses. The coloring method of $\operatorname{sign}\left(\lambda_{2}\right) \rho$ is given in Fig. 2. Each phenyl group is defined as a fragment in the IGM and IGMH calculations. The pink arrows indicate the defects in the IGM map.

Cyclo[18]carbon is a single ring system containing $18 s p$-hybridized carbon atoms. It was first experimentally observed in condensed phase in $2019,{ }^{[58]}$ since then it becomes one of the hottest 
molecules and received wide investigations, we have also conducted extensive theoretical studies on this molecule and its analogous. ${ }^{[4,59-66]}$ Recently, ultrafast supramolecular rotors composed of a cyclo[18]carbon molecule encapsulated by a [n]cycloparaphenylenes ([n]CPP) type of molecule were designed, ${ }^{[67]}$ and the authors employed IGM analysis via Multiwfn to visually study the intermolecular interaction within this host-guest complex. The complex [12]CPP: $\mathrm{C}_{18}$ is taken as instance here to compare the difference between IGM and IGMH. $\operatorname{sign}\left(\lambda_{2}\right) \rho$ colored $\delta g^{\text {inter }}$ isosurface maps are provided in Fig. 4, and in the IGMH map non-hydrogen atoms are colored by $\delta G^{\text {atom }}$. It is seen that the IGMH map nicely reveals the actual main interaction region between the guest and host molecules, the green color on the isosurface indicates that electron density in this region is very low, implying the interaction is fully dispersion dominated. Furthermore, the green and orange colors on some atoms of the [12]CPP molecule vividly highlight the atoms having major contribution to the host-guest complexation. The IGM map also correctly exhibited the intermolecular interaction, however there are misleading blue and orange colors on the IGM $\delta g^{\text {inter }}$ isosurface, mostly because the very thick isosurface has intruded into high density regions that are too close to nuclei. We noticed that this problem of IGM method has led to evidently incorrect conclusion from the authors: "The regions colored in blue, on the other hand, reflect a rather different type of interaction". ${ }^{[67]}$ In fact, there is only one type of interaction between the [12]CPP and cyclo[18]carbon, as revealed by the IGMH map, and which is also supported by the mapped color on the RDG isosurface in NCI map, see Fig. S2.
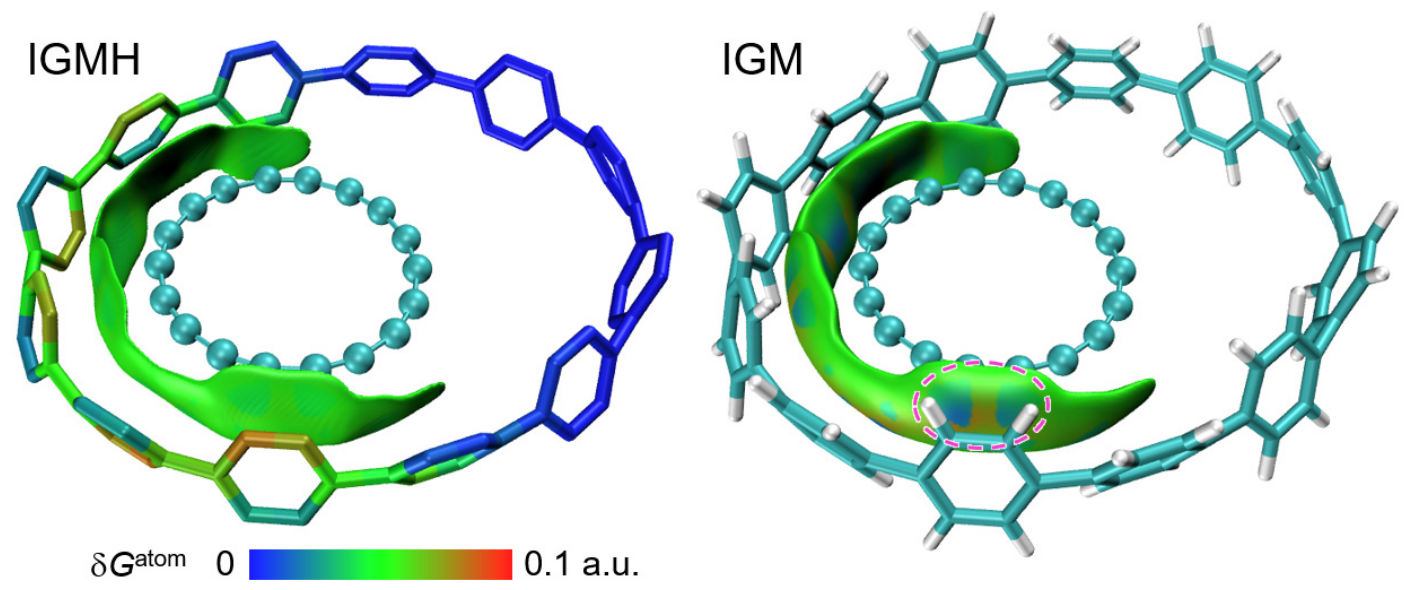

Fig. $4 \operatorname{sign}\left(\lambda_{2}\right) \rho$ colored isosurfaces of $\delta g^{\text {inter }}=0.002$ a.u. corresponding to IGMH and IGM analyses for [12]CPP: $\mathrm{C}_{18}$ complex. The [12]CPP and $\mathrm{C}_{18}$ molecules are defined as the two fragments. The coloring method of $\operatorname{sign}\left(\lambda_{2}\right) \rho$ is given in Fig. 2. In the IGMH map, atoms are colored by $\delta G^{\text {atom }}$ to highlight their relative contributions, while the hydrogens are hidden for clarity. The unreasonable coloring areas in IGM map is marked by pink circle.

Hydrogen bond (H-bond) ${ }^{[68]}$ and halogen bond (X-bond) ${ }^{[69,70]}$ are very common types of weak interaction and have been widely studied. In order to test whether IGMH can satisfactorily reveal these two important kinds of interactions, we performed IGMH analysis on adenine-thymine (AT) base pair and iodine substituted AT (I-AT). The latter was designed by Guerra et al. by replacing the two hydrogens that can form H-bond in AT with iodine atoms. ${ }^{[71]}$ The $\operatorname{sign}\left(\lambda_{2}\right) \rho$ colored IGMH $\delta g^{\text {inter }}$ isosurface maps for these systems are given in Fig. 5, and for comparison purpose, the 
corresponding IGM maps are also presented. From the IGMH maps, one can find that $\delta g^{\text {inter }}$ isosurface occurs in every expected region. For AT, the $\mathrm{N} \cdots \mathrm{H}-\mathrm{N}$ and $\mathrm{N}-\mathrm{H} \cdots \mathrm{O}$ H-bond interactions are clearly exhibited by elliptic $\delta g^{\text {inter }}$ isosurfaces with blue color in the central area, and since the blue on the former is evidently darker than the latter, one can easily judge that the $\mathrm{N} \cdots \mathrm{H}-\mathrm{N}$ interaction is relatively stronger because of its larger electron density in the interaction region. The $\mathrm{C}-\mathrm{H} \cdots \mathrm{O}$ interaction is also revealed by an IGMH $\delta g^{\text {inter }}$ isosurface, which has a tiny size and completely green color, indicating that this interaction is fairly weak, though not completely negligible. In the IGMH map of I-AT, it is seen that the notable X-bond between the iodine atom and nitrogen atom is distinctly unveiled by the blue region in the IGMH $\delta g^{\text {inter }}$ isosurface. Since the blue color is quite prominent, it can be inferred that the X-bond should be strong. In addition, the green region in the IGMH map of I-AT also vividly reveals the dispersion-dominated interaction between the iodine and carbon atoms, as well as between the two iodine atoms. The four largest $\delta G^{\text {pair }}(\%)$ values are labelled in the IGMH map of I-AT, the magnitude of the values is fully in line with the interatomic interaction strength that exhibited by the IGMH $\delta g^{\text {inter }}$ isosurfaces. For example, the $\delta G^{\text {pair }}(\%)$ corresponding to the strong $\mathrm{I} \cdots \mathrm{N}$ X-bond is by far larger than any other interaction. This observation demonstrated the practical value of $\delta G^{\text {pair }}(\%)$ index in quantitative comparison purpose. However, we still emphasize that $\delta G^{\text {pair }}(\%)$ should not be simply regarded as the percentage contribution to interfragment interaction energy. After all, the definition of $\delta G^{\text {pair }}(\%)$ is quite simple and does not take any energy-related factor into account. The IGM maps of AT and I-AT in Fig. 5, in our viewpoint, have an evidently poorer graphical effect than IGMH maps. The IGM $\delta g^{\text {inter }}$ isosurfaces representing the H-bonds and X-bond are bulgy and ugly, moreover, an evidently inappropriate coloring region is clearly visible on the IGM $\delta g^{\text {inter }}$ isosurface of I-AT, the red zone falsely indicates that there is a considerable steric repulsion effect between the carbon and iodine atoms.

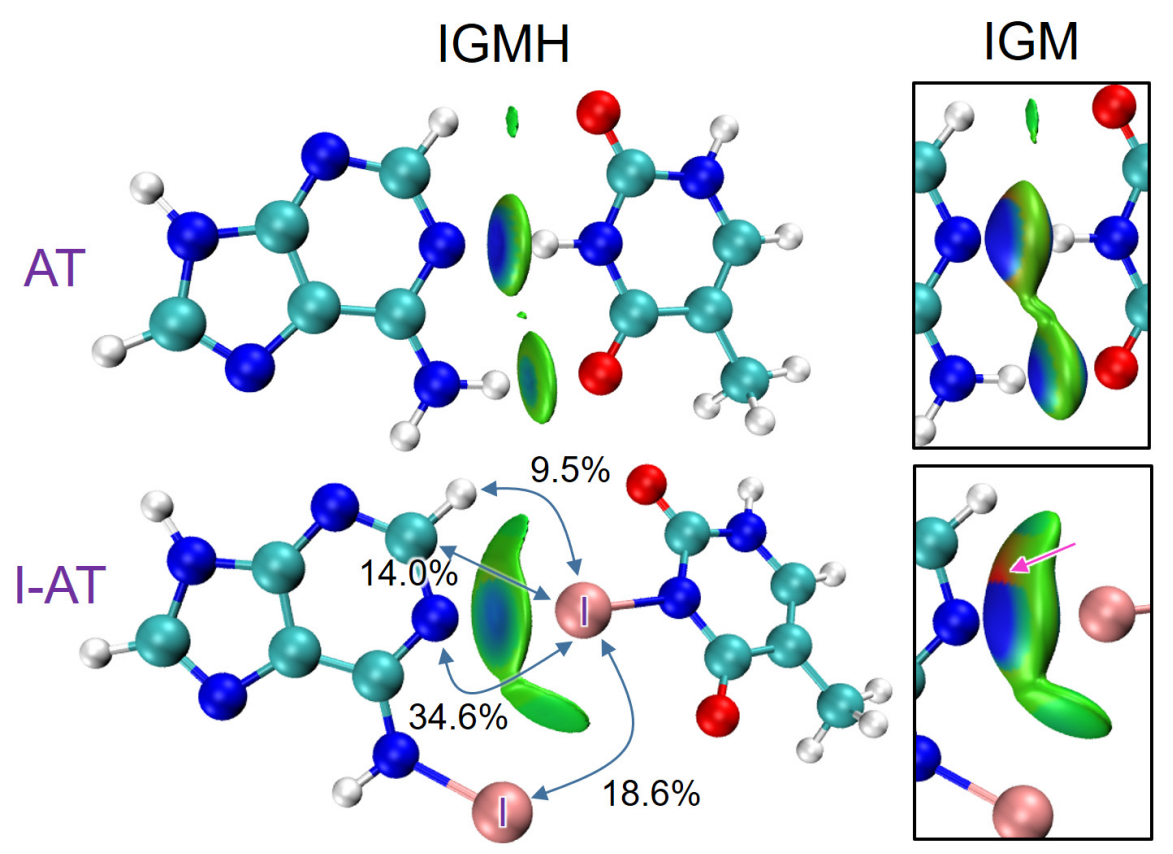

Fig. 5 (a) $\operatorname{sign}\left(\lambda_{2}\right) \rho$ colored isosurfaces of $\delta g^{\text {inter }}=0.006$ a.u. corresponding to IGMH and IGM analyses for adenine-thymine (AT) base pair and iodine substituted AT (I-AT). The coloring method of $\operatorname{sign}\left(\lambda_{2}\right) \rho$ is given in Fig. 2. In the map of I-AT, the largest four $\delta G^{\text {pair }}(\%)$ terms derived from IGMH analysis are 
labelled, and an evidently improper coloring area on the IGM $\delta g^{\text {inter }}$ isosurface is highlighted by a pink arrow.

In 2010, Cooper and coworkers reported synthesis of a series of triply interlocked covalent organic cages. ${ }^{[72]}$ They are dimer consisting of two tetrahedral monomeric cages, the two molecules cannot be dissociated without cleavage of a chemical bond. This kind of interesting system is ideal for illustrating the capability of IGMH to reveal intermolecular interaction in complicated complexes. The $\mathbf{3 b}$ system reported in their work is taken as an instance here. The structure determined by X-ray diffraction experiment was adopted but hydrogens were optimized by us to refine their positions. Fig. 6(a) presents $\operatorname{sign}\left(\lambda_{2}\right) \rho$ colored IGMH $\delta g^{\text {inter }}$ isosurfaces of 0.004 a.u., the two monomers are respectively defined as the two fragments. Another perspective of this figure is provided in Fig. S3. It can be clearly seen that all intermolecular interactions are clearly revealed by the IGMH map, notable interactions occur in many contact areas. Since the isosurfaces are all green, all the interactions should be dominated by dispersion effects, whose strength is known to be weak. It is worth noting that at center of the dimer, there is an evident $\pi-\pi$ stacking region, as there exists a flat and broad $\delta g^{\text {inter }}$ isosurface. This stacking interaction was also inferred by Cooper et al. ${ }^{[72]}$ according to dimer structure. We also plotted IGM isosurface map in Fig. S4, it can be seen that again there are some areas on the isosurfaces showing spurious blue or red color, which may make researchers mistakenly believe that there are relatively strong attraction or steric hindrance interactions between the two molecules. $\delta G^{\text {atom }}$ colored structure map of $\mathbf{3 b}$ is given in Fig. $6(\mathrm{~b})$, from which the contribution from each atom to the intermolecular interaction can be very intuitively examined. According to the color bar, the redder (bluer) the color, the larger (smaller) the atomic contribution to the complexation between the two monomers. It can be vividly seen that the atoms participating in the $\pi-\pi$ stacking contribute most, and the contributions from other atoms around the center of the dimer are also prominent. Since the atoms at the top and bottom of the dimer are far away from the atoms in another molecule, their contributions to the binding are basically negligible.
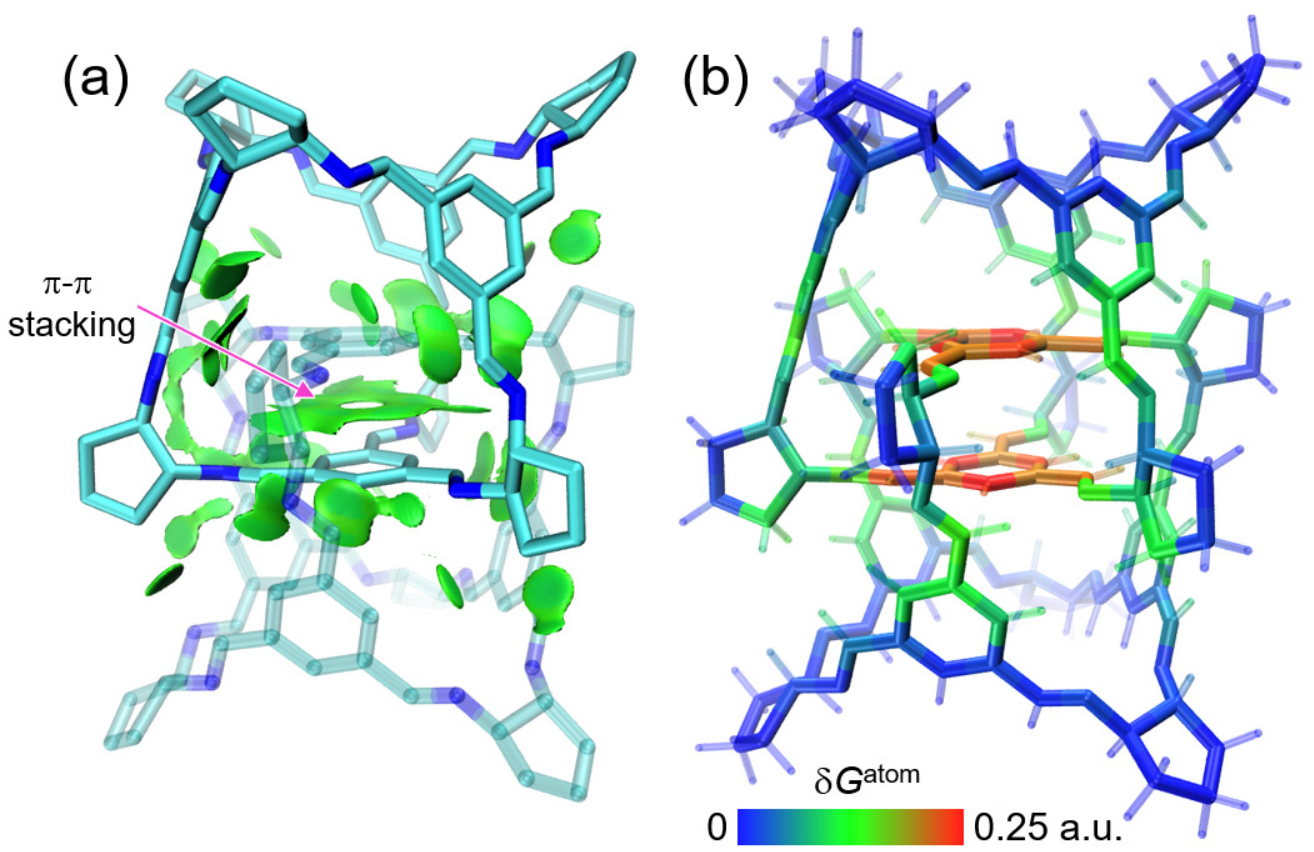

Fig. 6 IGMH maps of $\mathbf{3 b}$. The two monomers are respectively defined as the two fragments. (a) $\operatorname{sign}\left(\lambda_{2}\right) \rho$ 
colored isosurfaces of $\delta g^{\text {inter }}=0.004$ a.u. The coloring method of $\operatorname{sign}\left(\lambda_{2}\right) \rho$ is given in Fig. 2 . To make it easier to distinguish the two molecules, they are plotted using opacity and transparent styles respectively. For clarity the hydrogens are not shown (b) $\delta G^{\text {atom }}$ colored dimer structure. Hydrogens are drawn transparently. The redder (bluer) the color, the larger the contribution of the atom to intermolecular interaction.

Transition metal surface is able to chemically or physically adsorb small molecules. Grimme et al. studied physical adsorption of benzene molecule on $\mathrm{Ag}(111)$ surface using cluster model. ${ }^{[73]}$ We optimized the same system using PBE0-D3(BJ) functional ${ }^{[74]}$ in combination with def2-SV(P) basis set, ${ }^{[75]}$ which are then subjected to IGM and IGMH analyses to graphically reveal the interaction between the adsorbed benzene and $\mathrm{Ag}$ surface. The $\operatorname{sign}\left(\lambda_{2}\right) \rho$ colored $\delta g^{\text {inter }}$ isosurface maps are given in Fig. 7. In the IGM and IGMH calculations the benzene and Ag slab are defined as two fragments. From the green isosurface between the molecule and the slab in the IGMH map, it is evident that IGMH analysis nicely reveals the broad interaction region and reflects the true nature of the physical adsorption. It is noteworthy that by using Multiwfn in combination with VMD, the BCPs and bond paths derived from topology analysis module in Multiwfn can be easily appended to the IGMH map, and thus providing richer information to readers. In Fig. 7, the BCPs and bond paths corresponding to the benzene $\cdots \mathrm{Ag}$ interaction are drawn as orange spheres and brown lines, respectively. It can be seen that BCPs are almost exactly lying on the thin IGMH $\delta g^{\text {inter }}$ isosurface, which is a phenomenon that we commonly observed in actual studies. Obviously, the BCPs cannot reveal interaction regions as sufficient as IGMH analysis, however, as BCPs usually correspond to the most representative positions in interaction regions, properties of BCPs are useful in quantitative analysis. The electron density and energy density $(H)$ of one of the BCPs characterizing the benzene $\cdots \mathrm{Ag}$ interaction are provided in the IGMH map, the very low electron density ( $\rho^{\mathrm{BCP}}=0.013$ a.u.) directly corresponds to the green color of the $\delta g^{\text {inter }}$ isosurface, and the positive $H$ at the BCP reflects that the interaction has a non-covalent nature. ${ }^{[76]}$ The $\mathrm{Ag}$ atoms in the IGMH map of Fig. 7 are colored according to $\delta G^{\text {atom }}(\%)$ to exhibit their contributions to the adsorption interaction, the colors very vividly show that the Ag atoms at the bottom of the benzene have significant contribution; in particular, the three greenest atoms contribute most, this may be why they are just the three atoms directly connected by the three bond paths in the figure. Compared to the IGMH map, the IGM map in Fig. 7 is not so ideal; there are orange and blue colors on the very bulgy $\delta g^{\text {inter }}$ isosurface, which seriously interfere with the judgment of the actual nature of the interaction, and we found this problem cannot be suppressed by simply adjusting isovalue. In order to illustrate the advantage of IGMH over the popular NCI method, the $\operatorname{sign}\left(\lambda_{2}\right) \rho$ colored RDG isosurface is also provided in Fig. 7. The color and shape of the isosurface representing the adsorption interaction in the NCI map is very similar with that in IGMH map; however, as NCI analysis is unable to strictly separate intrafragment and interfragment interactions, enormous RDG isosurfaces showing metallic bond interactions appear among the Ag atoms, they evidently hinder the graphical display of the adsorption interaction. Although sometimes the undesirable RDG isosurfaces can be partially eliminated by some special treatments, such as properly defining the spatial range of grid data calculation, and screening the grid data of RDG via grid processing function in Multiwfn, it is obvious that they will complicate the calculation process. 


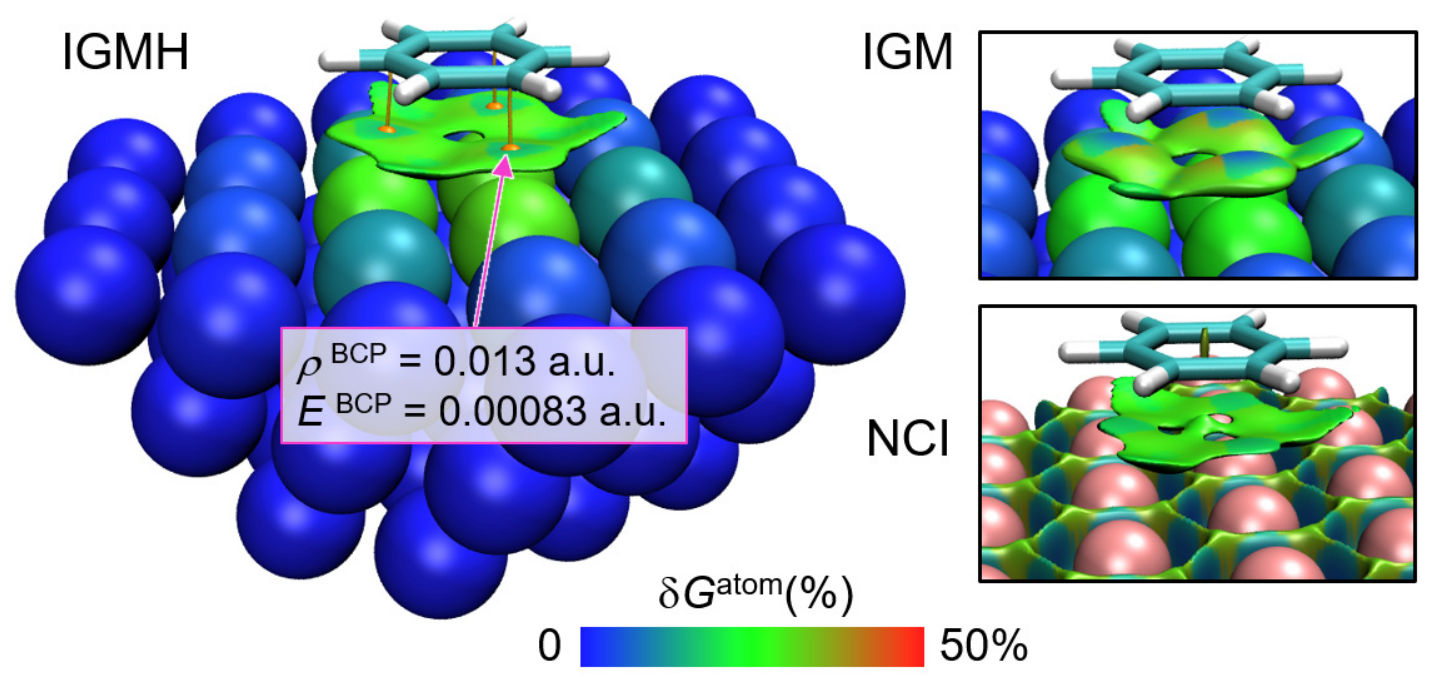

Fig. $7 \operatorname{sign}\left(\lambda_{2}\right) \rho$ colored IGMH $\delta g^{\text {inter }}=0.005$ a.u., IGM $\delta g^{\text {inter }}=0.007$ a.u. and RDG $=0.4$ isosurfaces for representing physical adsorption of benzene on $\operatorname{Ag}(111)$ surface. The coloring method of $\operatorname{sign}\left(\lambda_{2}\right) \rho$ is given in Fig. 2. In IGM and IGMH analyses the benzene and slab are respectively defined as the two fragments, and $\mathrm{Ag}$ atoms are colored according to $\delta G^{\text {atom}}(\%)$. In the IGMH map, bond critical points (orange spheres) and bond paths (brown lines) corresponding to benzene $\cdots$ Ag interaction are also shown.

The binding mode between ligands and proteins is a matter of great concern in the fields of molecular biochemistry and medicinal chemistry. Undoubtedly, graphically revealing the interactions is of great significance for deepening understanding of their binding mechanism and targeted design of more effective drug molecules. Here we employ the IGMH analysis to study the interaction between benzamidine and trypsin. The crystal structure of the protein-ligand complex was provided by Ref. [77] with PDB ID of 3ATL. Since the direct calculation of the entire complex using DFT far exceeds the currently affordable computational cost, we employ cluster model for this research. Specifically, a cluster composed of the benzamidine ligand and all amino acid residues within $3.5 \AA$ of it were extracted and then appropriately hydrogenated. In the subsequent geometry optimization, non-hydrogen atoms were fixed at the crystal coordinates while only hydrogen atoms were optimized. The cluster contains merely 158 atoms, so DFT calculation and IGMH analysis can be performed very easily. The $\operatorname{sign}\left(\lambda_{2}\right) \rho$ colored IGMH $\delta g^{\text {inter }}$ isosurface map of the cluster is given in Fig. 8, the ligand and the surrounding residues are defined as the two fragments in the analysis. In Fig. 8(a), a relatively large isovalue 0.006 a.u. is used, in this case very prominent intermolecular interactions can be viewed, including the four $\mathrm{N}-\mathrm{H} \cdots \mathrm{O}$ type of $\mathrm{H}$-bonds between the ligand and the protein, as highlighted by the pink arrows, as well as some dispersion-dominated interaction regions, as indicated by the brown arrows. Lowering the isovalue of $\delta g^{\text {inter }}$ can make regions corresponding to insignificant interactions also visible. In Fig. 8(b) the isovalue is reduced to 0.002 a.u., in this situation wide green isosurfaces occur above and below the benzamidine molecule, rendering that there are also nonnegligible dispersion interactions between the molecule and the amino acids above and below it. Fig. 8(c) presents NCI map, namely $\operatorname{sign}\left(\lambda_{2}\right) \rho$ colored isosurfaces of RDG $=0.5$. This map not only shows the interactions between the ligand and the protein, but also displays the interactions among the amino acid residues, thus the image looks very messy and difficult to analyze the ligand-protein interaction clearly. This map also shows a noteworthy shortcoming of NCI compared to IGMH, that is, NCI map has a high requirement on fineness of grid. In the current 
study, although the employed grid spacing of $0.15 \mathrm{Bohr}$ is already fairly fine, there are still many ugly jagged edges in the RDG isosurfaces, while the $\delta g^{\text {inter }}$ isosurfaces do not suffer from this problem. Further reducing the grid spacing can effectively reduce the jagged edges in the NCI map, unfortunately, it will significantly increase computational cost and file size of grid data.

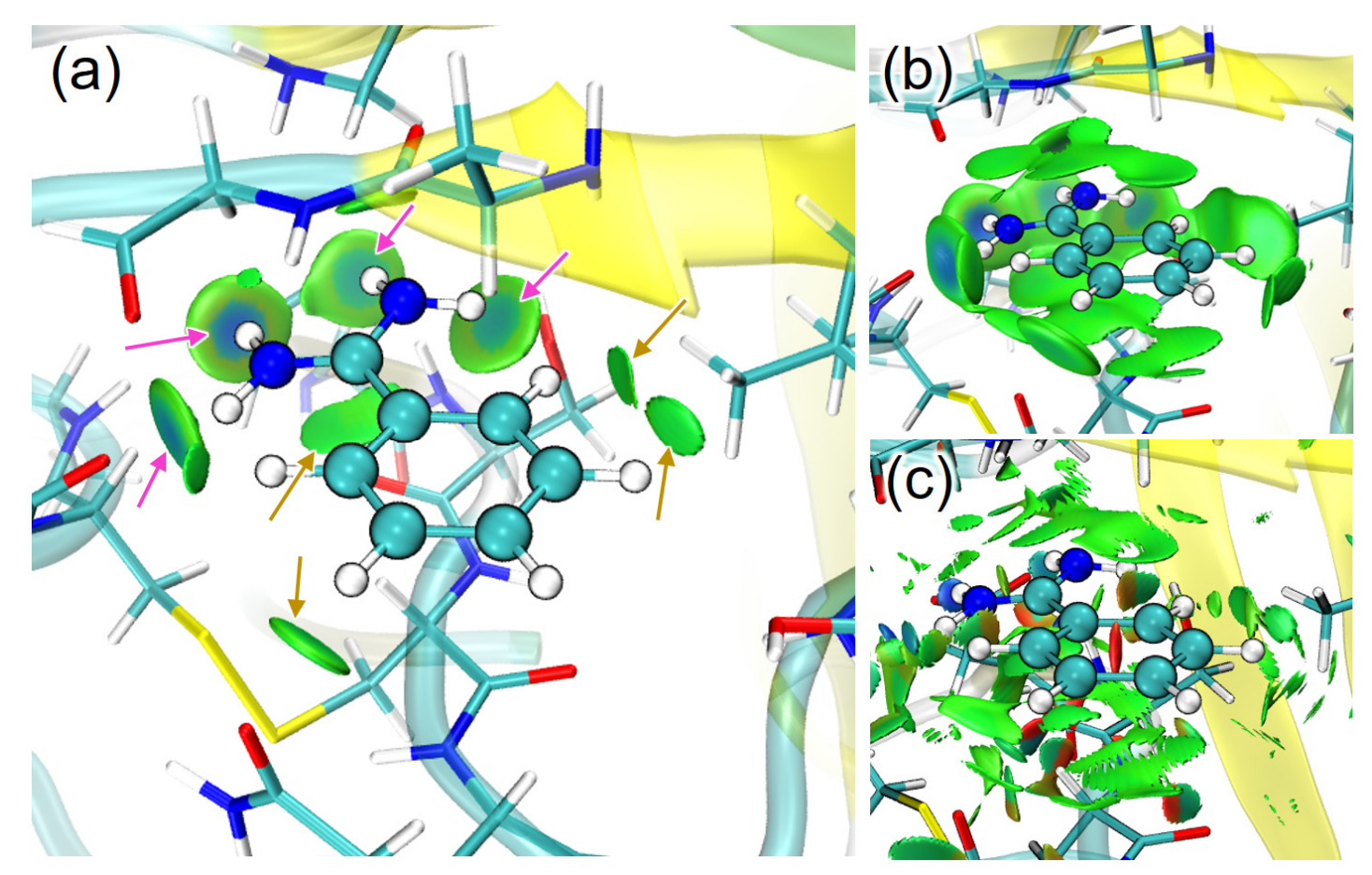

Fig. 8 Cluster model consisting of benzamidine ligand (ball and stick style) and the amino acid residues in trypsin closely contacted to it (stick style) in protein-ligand complex (PDB ID: 3ATL). Backbone of the protein is plotted in new cartoon style with transparent material. (a) and (b): $\operatorname{sign}\left(\lambda_{2}\right) \rho$ colored IGMH $\delta g^{\text {inter }}=0.006$ and 0.002 a.u. isosurfaces, respectively. The coloring method of $\operatorname{sign}\left(\lambda_{2}\right) \rho$ is given in Fig. 2. Pink and brown arrows highlight hydrogen bonds and dispersion-dominated interaction regions, respectively. (c) $\operatorname{sign}\left(\lambda_{2}\right) \rho$ colored of $\mathrm{RDG}=0.5$ isosurface in $\mathrm{NCI}$ analysis, the coloring method is the same as Fig. S2.

\subsection{Revealing weak interactions in periodic systems}

Originally Multiwfn only supports analysis for isolated systems, while recently we have largely extended its application range to periodic systems. Currently NCI, IRI, IGM and IGMH analyses have fully supported consideration of periodic boundary condition. For NCI, IRI and IGMH analysis, currently the wavefunction generated by periodic DFT calculation of the free and very fast CP2K code $^{[47]}$ is acceptable. In this section, we provide a few examples to illustrate the great value of applying IGMH method to reveal weak interactions in periodic systems.

Ice is the most important molecular crystal, and it is well-known that each water molecule in ice forms multiple $\mathrm{H}$-bonds with surrounding waters. In order to graphically reveal the H-bonds, we constructed a $2 \times 2 \times 2$ supercell based on experimental crystal structure of ice in $I_{h}$ form, ${ }^{[78]}$ then optimized hydrogens while fixing positions of oxygens. In the IGMH analysis, a water molecule at the center of the supercell and all other waters are respectively defined as the two fragments. The 
resulting $\operatorname{sign}\left(\lambda_{2}\right) \rho$ colored IGMH $\delta g^{\text {inter }}=0.03$ a.u. isosurface map is shown in Fig. 9. As can be seen, the four H-bonds formed between the central water molecule and others are very clearly shown. Since the blue color on the isosurfaces is relatively dark, it can be anticipated that the H-bond interactions in ice should be relatively strong and stable.

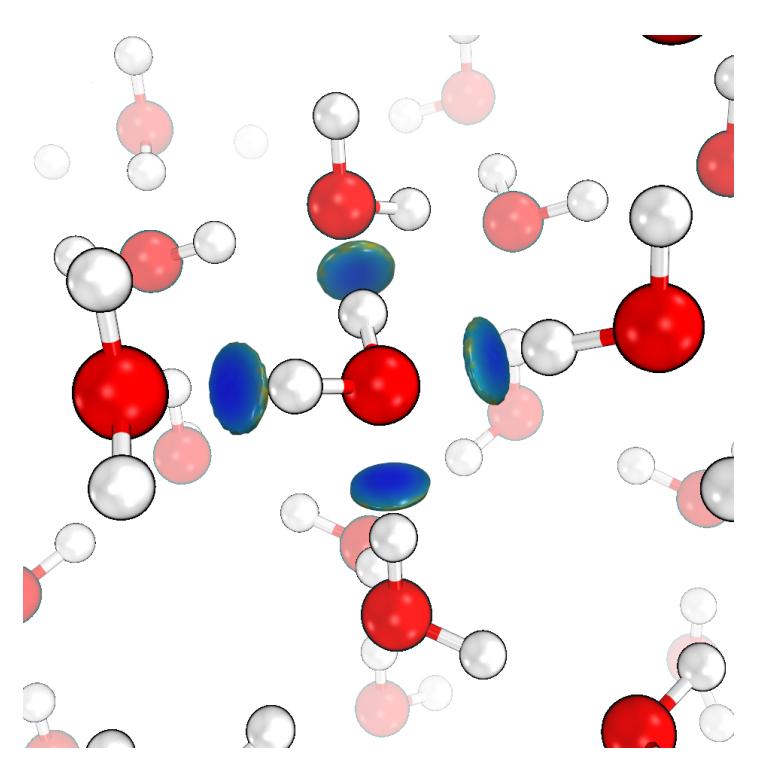

Fig. $9 \operatorname{sign}\left(\lambda_{2}\right) \rho$ colored IGMH $\delta g^{\text {inter }}=0.03$ a.u. isosurfaces of ice crystal of $I_{h}$ form. The coloring method of $\operatorname{sign}\left(\lambda_{2}\right) \rho$ is given in Fig. 2. The water molecule at the center of this figure and all other waters are defined as the two fragments in the IGMH analysis.

Zeolite is a very representative porous material with strong ability to physically adsorb small molecules. Graphical display of the interaction between zeolite and adsorbate is undoubtedly an important help for the in-depth study of the adsorption mechanism and adsorption capacity of zeolite. As an example, we manually put a toluene into cavity of HEU type of zeolite ${ }^{[79]}$ and optimized geometry of the adsorption system, then IGMH map is plotted by defining the toluene and zeolite as the two fragments, see Fig. 10. It can be seen that the isosurface map is very ideal, the isosurfaces elegantly reveal the regions where interaction between the zeolite and toluene is most prominent. Since the isosurfaces are green everywhere, it shows that the adsorption of toluene by zeolite mainly comes from weak dispersion attraction. 


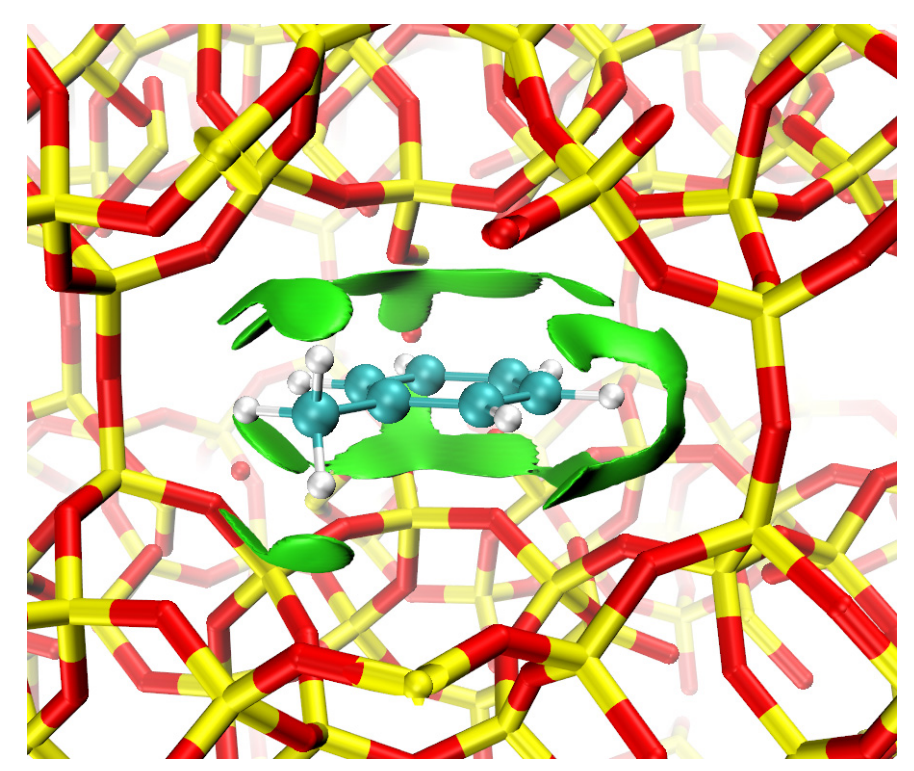

Fig. $10 \operatorname{sign}\left(\lambda_{2}\right) \rho$ colored IGMH $\delta g^{\text {inter }}=0.005$ a.u. isosurfaces of adsorbed toluene in zeolite cavity. The coloring method of $\operatorname{sign}\left(\lambda_{2}\right) \rho$ is given in Fig. 2. Red and yellow atoms correspond to oxygen and silicon, respectively. The toluene molecule and zeolite are defined as the two fragments in the IGMH analysis.

We also applied IGMH analysis on a porous covalent organic framework (COF) with twodimensional layered character to try to reveal the interlayer interaction. The crystal structure was taken from CURATED covalent organic frameworks database (ID: 12000N2) ${ }^{[80]}$ positions of hydrogens are then optimized by us via DFT calculation. The corresponding IGMH map is given in Fig. 11, the two layers of COF in the cell are defined as the two fragments in the analysis. As can be seen, there is an infinitely extended green isosurface between the atoms in the two layers, which clearly shows the prominent interlayer $\pi-\pi$ stacking interaction. It is noteworthy that when we calculating data for plotting this map via Multiwfn, the periodicity in the direction perpendicular to the layers is ignored for clarity. If full three-dimensional periodicity is considered in the IGMH analysis, the $\delta g^{\text {inter }}$ isosurfaces showing interactions between the two layers in the current cell and the layers in adjacent image cells will also be visible, as shown in Fig. S5.

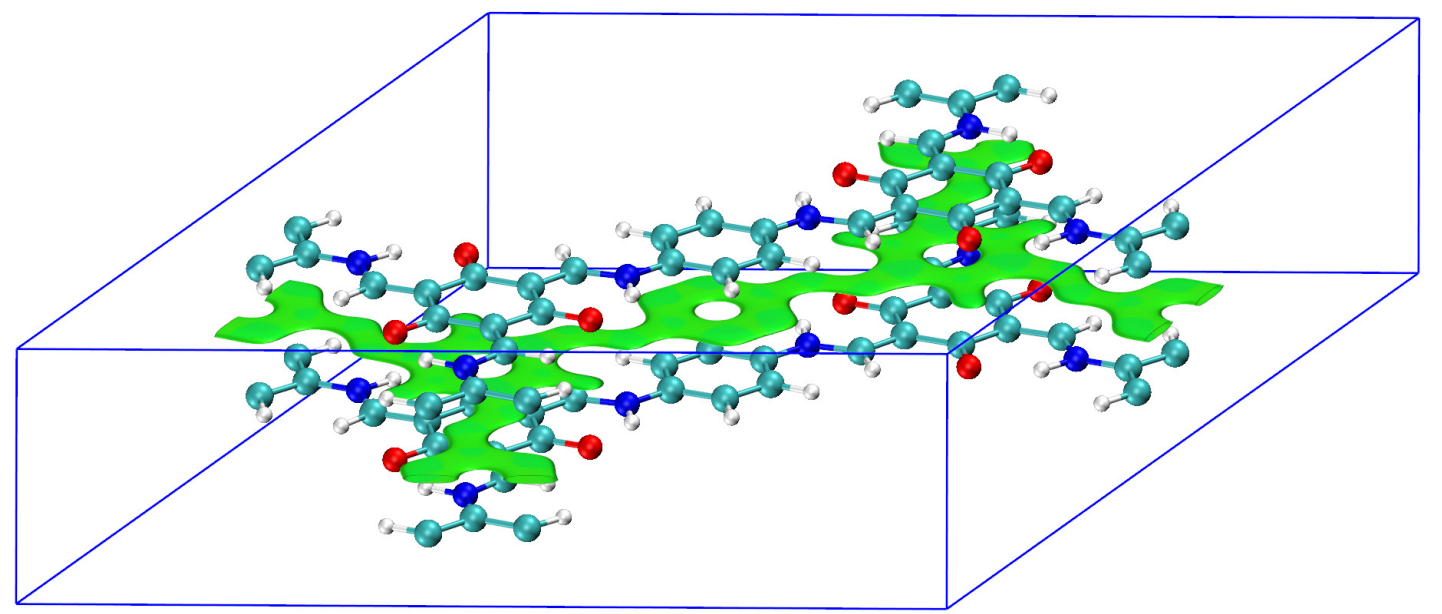

Fig. $11 \operatorname{sign}\left(\lambda_{2}\right) \rho$ colored IGMH $\delta g^{\text {inter }}=0.004$ a.u. isosurfaces of a representative covalent organic framework system. The upper and lower layers are defined as the two fragments in the IGMH analysis. 
The coloring method of $\operatorname{sign}\left(\lambda_{2}\right) \rho$ is given in Fig. 2. The blue box displays the cell used in periodic DFT calculation. For clarity, the periodicity in the direction perpendicular to the layers is ignored in the IGMH analysis.

\subsection{Revealing strong interactions}

IGMH can not only reveal weak interactions as shown above but also reveal strong interactions like chemical bond bonds. In this section, we will present a few instances to illustrate this point.

Crown ethers can stably bind alkali cations via very strong electrostatic attraction by its electronegative oxygen atoms. Here we take a complex formed by a crown ether derivative and a $\mathrm{Li}^{+}$cation as instance to examine the ability of IGMH method in revealing their interactions, and meantime illustrate its difference with respect to NCI method. The IGMH and NCI maps with properly selected isovalues are given in Fig. 12. BCPs and bond paths between the cation and the molecule are generated by Multiwfn and plotted together in the IGMH map. It can be seen from the figure that the isosurface of $\delta g^{\text {inter }}$ defined by IGMH clearly exhibits the interaction between the $\mathrm{Li}^{+}$ cation and all five surrounding oxygen atoms, the light blue color areas on the isosurface indicate that electron density in these regions is notably larger than common dispersion interaction regions. Electron density values at the BCPs corresponding to these interactions are also shown in the figure, such a combination of AIM topology analysis and IGMH analysis facilitates quantitative investigation and discussion, and largely avoids the visual arbitrariness due to plotting settings such as choice of color scale and lighting. The NCI isosurface around the $\mathrm{Li}^{+}$cation in Fig. 12 also correctly represents the $\mathrm{Li}^{+} \cdots \mathrm{O}$ interactions, however, the isosurfaces exhibiting $\mathrm{O} \cdots \mathrm{O}$ interactions are completely merged with it, and meantime the isosurfaces corresponding to the $\mathrm{H} \cdots \mathrm{H}$ interactions and to the steric effect in the aromatic ring are also visible, these uninterested isosurfaces in the figure obviously interfere with visual investigation of the interfragment interaction of real interest. This problem is more severe for more complex systems, such as the ligand-protein complex shown earlier.
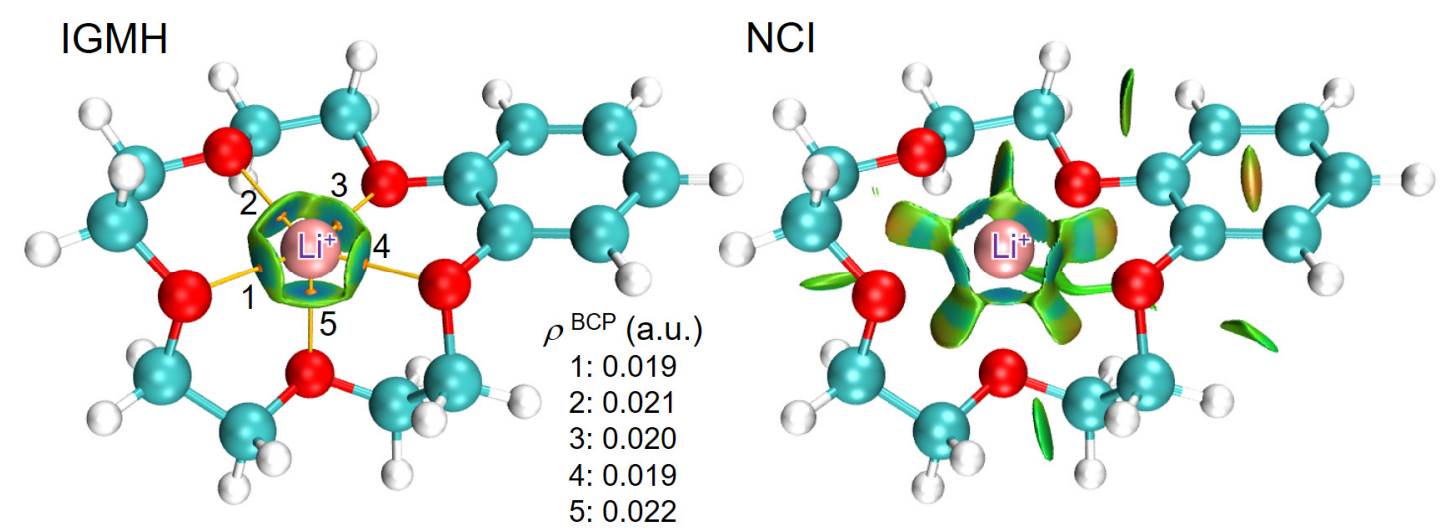

Fig. $12 \operatorname{sign}\left(\lambda_{2}\right) \rho$ colored IGMH $\delta g^{\text {inter }}=0.01$ a.u. and $\mathrm{RDG}=0.6$ isosurfaces of complex formed by a crown ether derivative and $\mathrm{Li}^{+}$cation. The coloring method of $\operatorname{sign}\left(\lambda_{2}\right) \rho$ is the same as Fig. 2 , but lower and upper limits are changed to -0.4 and 0.4 respectively to make colors more vivid. Orange spheres and yellow lines in the IGMH map are BCPs and bond paths, respectively. Electron density values at the BCPs are indicated by texts. 
Tetra(trimethylsilyl)tetrahedrane is a molecule yielded by substituting the four hydrogens in tetrahedrane with trimethylsilyl groups, its structure map corresponding to superposition of atomic van der Waals spheres is shown in Fig. 13. It can be seen that this system is very crowded and there should be notable vdW interactions between each pair of trimethylsilyl group. In this example, we define the four trimethylsilyl groups and the tetrahedral $\mathrm{C}_{4}$ core as the five fragments in IGMH analysis, the resulting maps are given in Fig. 13. The $\delta g^{\text {inter }}$ isosurfaces at a relatively large isovalue, 0.05 a.u., clearly exhibits the strong interactions between the $\mathrm{C}_{4}$ core and trimethylsilyl groups, namely the four C-Si covalent bonds. If isovalue of $\delta g^{\text {inter }}$ is reduced to a small value 0.003 a.u. so that much weaker interactions can also be revealed, the isosurfaces corresponding to the evident $\mathrm{vdW}$ interactions between each pair of trimethylsilyl group will be visible, as shown in Fig. 13; however at this time the isosurfaces corresponding to the $\mathrm{C}$-Si bonds become too swollen and not easy to visually inspect. $\delta g^{\text {intra }}$ is complementary to $\delta g^{\text {inter }}$ and focus on exhibiting intrafragment interactions. As can be seen in the $\delta g^{\text {intra }}$ map in Fig. 13, the C-H bonds and C-Si bond of each trimethylsilyl group fragment, as well the four $\mathrm{C}-\mathrm{C}$ bonds in the $\mathrm{C}_{4}$ core fragment, are all vividly revealed by the $\delta g^{\text {intra }}$ isosurfaces of 0.1 a.u. It is worth mentioning that if practical purpose is to reveal all kinds of interactions in the system by a single map, and there is no need to divide fragments to individually study the interactions within or between specific fragments, using IRI analysis is a better and more convenient choice. As shown in Fig. S6, the isosurfaces of IRI $=0.7$ a.u. exhibit all chemical bonds and weak interactions simultaneously and satisfactorily.

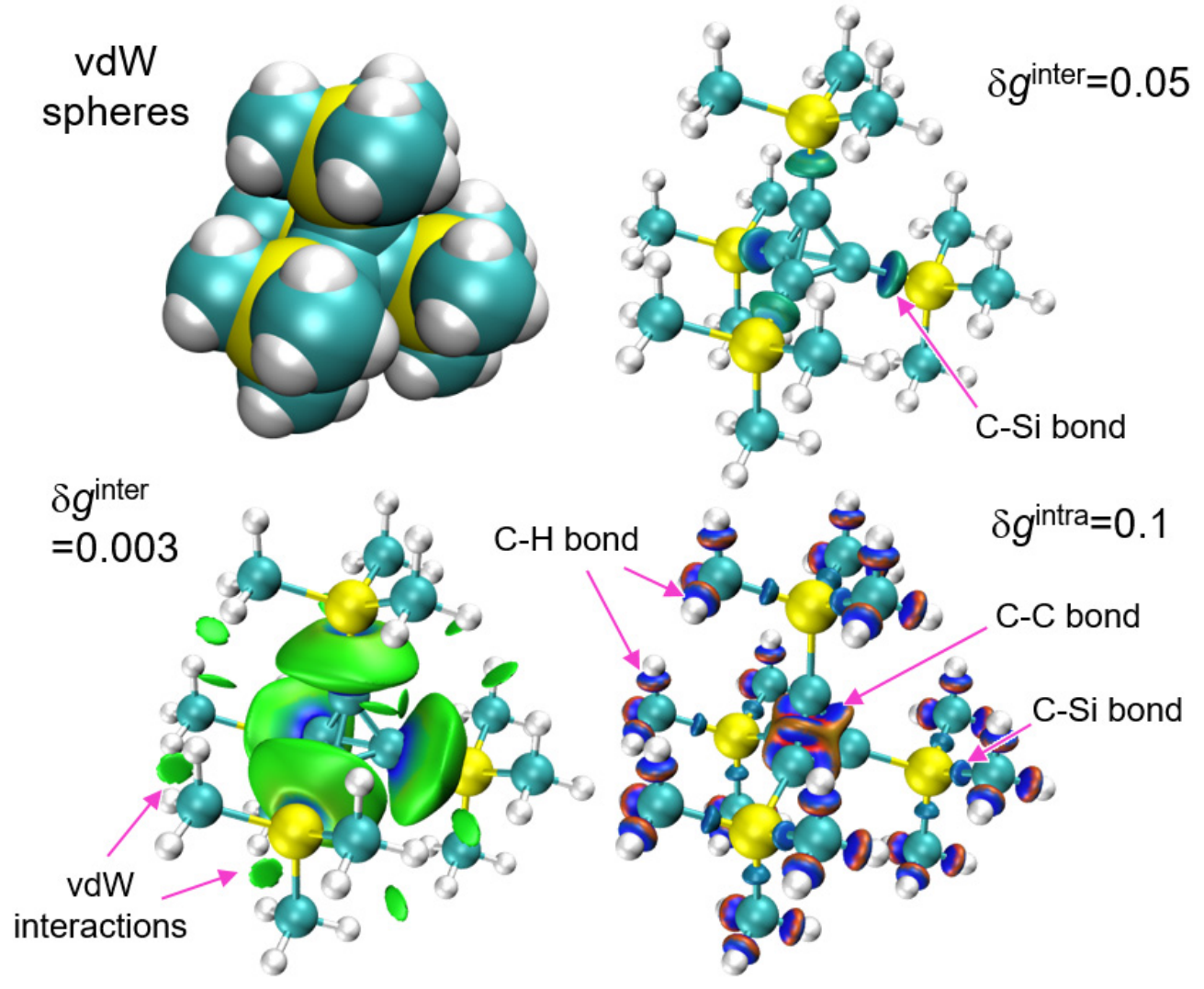

Fig. 13 Molecular structure map (vdW style), as well as $\operatorname{sign}\left(\lambda_{2}\right) \rho$ colored IGMH $\delta g^{\text {inter }}$ and $\delta g^{\text {intra }}$ maps of tetra(trimethylsilyl)tetrahedrane. The coloring method of $\operatorname{sign}\left(\lambda_{2}\right) \rho$ is the same as Fig. 2, but lower and upper limits are changed to -0.2 and 0.2 a.u. respectively for better graphical effect. 
Finally, we employ IGMH analysis on two representative atomic clusters, tetrahedral $\mathrm{P}_{4}$ and planar $\mathrm{Li}_{6}$, to further evaluate its ability in revealing interactions in relatively unusual systems. For comparison, the IGM and IRI analyses, as well as the valence electron density analysis that we discussed in depth in Ref. [81], are also taken into account. The plotted maps are collectively given in Fig. 14, the isovalues of the isosurfaces were properly chosen to best represent interactions in the systems. Since in the present study there is no need to partition fragments, in the IGMH and IGM analyses we directly examine the $\delta g$ function, which is equivalent to $\delta g^{\text {intra }}$ when defining the whole system as the only fragment in Multiwfn. From the isosurfaces of $\delta g$ in the IGMH map of $\mathrm{P}_{4}$, it is found that there is a blue area in the isosurface between each pair of phosphorus atoms, which unambiguously shows that there is a chemical bond, and it possesses a much larger electron density than weak interaction regions. This observation reflects the covalent characteristics of the P-P bond, as formation of a covalent bond is always companied by accumulation of electron density in bonding region. ${ }^{[37]}$ The red areas in the IGMH map also well exhibit the steric hindrance in each threemembered ring and that in the cage. The IGM map of $\mathrm{P}_{4}$ has an obviously poorer graphical effect than the IGMH map; in particular, the positions of the blue isosurfaces corresponding to the P-P bonds are unreasonable. The centroids of the blue isosurfaces in the IGM map are obviously biased to the cage center relative to the bond axis, which incorrectly indicate the actual positions of the P$\mathrm{P}$ bonds. This problem of IGM should come from the fact that it does not take actual electronic structure into account at all. Although the underlying idea of the IRI method to reveal interaction regions is quite different from that of IGMH, the information conveyed by the IRI map for the $\mathrm{P}_{4}$ cluster is very similar to the IGMH map, namely it also reasonably shows the existence of the P-P bonds and steric hindrance in the rings and cage.
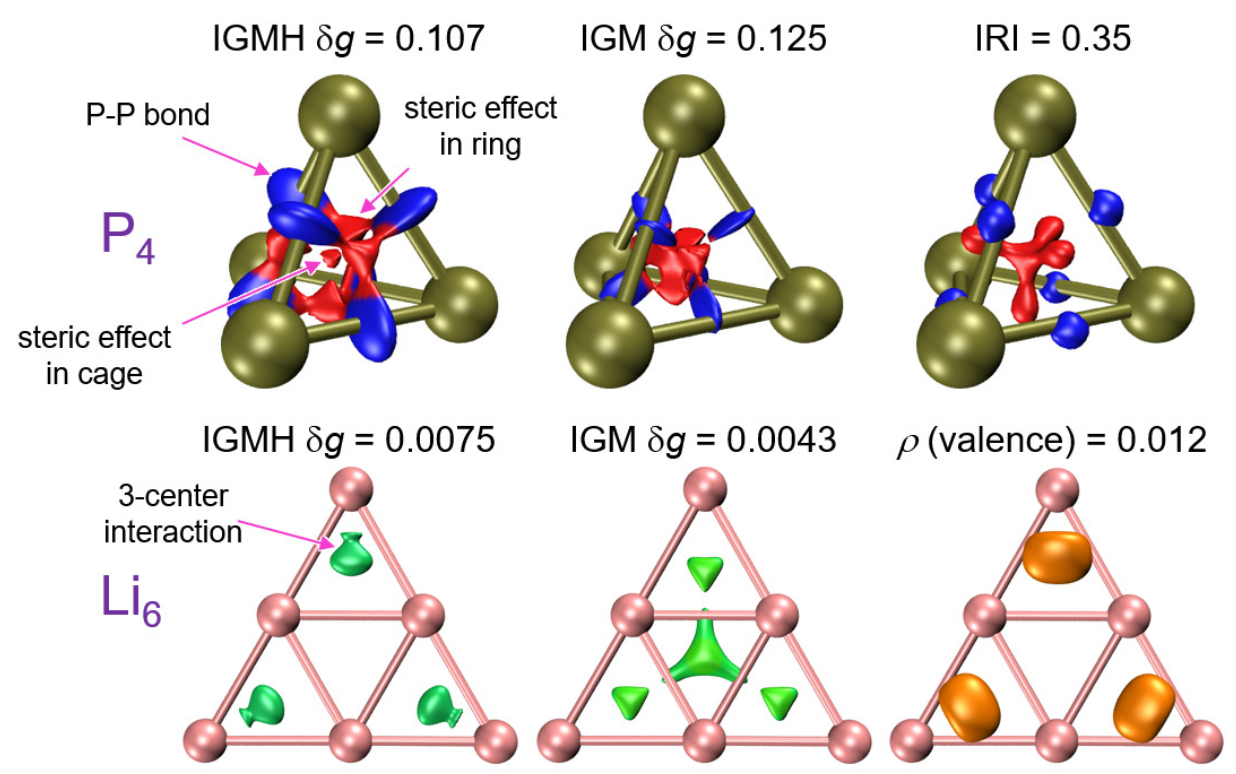

Fig. 14 Various maps of $\mathrm{P}_{4}$ and $\mathrm{Li}_{6}$ atomic clusters, including $\operatorname{sign}\left(\lambda_{2}\right) \rho$ colored IGMH $\delta g$ and IGM $\delta g$ isosurfaces, $\operatorname{sign}\left(\lambda_{2}\right) \rho$ colored IRI isosurfaces, and isosurface of valence electron density. The coloring method of $\operatorname{sign}\left(\lambda_{2}\right) \rho$ is the same as Fig. 2. All units in the labels are a.u.

The planar triangular $\mathrm{Li}_{6}$ cluster contains evident three-center two-electron (3c-2e) interactions, 
this can be demonstrated via different ways, such as ELF, ${ }^{[43,44]}$ multi-center index,${ }^{[82,83]}$ deformation density, ${ }^{[81]}$ orbital localization, ${ }^{[84]}$ and even the very simple valence electron density, $\rho$ (valence) ${ }^{[81]}$ The $\rho$ (valence) denotes the electron density without contribution of core orbitals. The isosurface map of $\rho$ (valence) of $\mathrm{Li}_{6}$ in Fig. 14 clearly indicates that there is an obvious three-center interaction between the three $\mathrm{Li}$ atoms at each corner, which results in relatively large electron density in the corresponding region. Can this multi-center interaction also be revealed by IGMH analysis? The answer is yes. As can be seen from Fig. 14, the distribution of IGMH $\delta \mathrm{g}$ between the three Li atoms at each corner is obviously greater than that between the three Li atoms in the center of the system, faithfully reflecting the existence of the three-center shared electron interaction. In contrast, the $\delta g$ defined by IGM does not have the same capacity to correctly identify the multi-center interactions in different regions. Fig. 14 shows that the IGM $\delta \mathrm{g}$ is also large in the center of the cluster, thus it may make researchers mistakenly believe that there is also a significant three-center interaction. This shortcoming of IGM is naturally expected since multicenter interaction purely stems from electronic effect rather than structural effect, and its occurrence is always accompanied by a considerable change in electronic distribution relative to promolecular state, hence revealing multicenter interactions without consideration of actual electronic structure is in principle impossible.

\section{Conclusion and remarks}

IGM is a very flexible and powerful method for graphically studying various kinds of interactions in chemical systems. In this article, we proposed a new variant of IGM, named IGMH, which is defined based on actual electron density and has a more rigorous physical basis than the original IGM method. As can be seen from the abundant application examples, IGMH is able to ideally reveal weak interactions and chemical bond interactions in a wide variety of chemical systems. Compared with IGM, the graphical effect of IGMH method is often significantly better in revealing weak interactions. The isosurfaces in IGMH map are not only less bulgy and thus look clearer than those in IGM map, but also the frequently observed misleading coloring on the IGM isosurfaces is fully avoided. Moreover, IGMH has a much better capacity than IGM in revealing chemical bond interactions since actual electron density is involved in the definition of IGMH.

Compared to the already very popular NCI method, a key advantage of IGMH is that fragments can be defined, so as to strictly separate interfragment and intrafragment interactions. This feature makes visual analysis much easier for systems with complex three-dimensional structural character. Another important advantage is that there are many quantitative indices available under the framework of IGMH, which are very valuable in identifying important contributions of various atoms and atomic pairs to interfragment interactions. An additional noteworthy advantage is that the isosurfaces in IGMH map are often less jaggy than those in NCI map when grid quality is not extremely fine. Of course, increasing number of grid points in plotting NCI map can effectively alleviate the jaggy problem, but the cost will increase considerably, especially for relatively large systems.

IGMH has been supported recently by Multiwfn. In this program, the IGMH analysis module is very flexible, computationally efficient, easy-to-use, and supports a wide range of input file formats. This analysis can not only be applied to molecular systems but also to periodic systems. In 
order to facilitate readers to quickly learn how to realize the IGMH analysis via Multiwfn, we have prepared a detailed tutorial and highly welcome readers to check: http://sobereva.com/multiwfn/res/IGMH tutorial.zip. We hope that IGMH will become a new popular visual analysis method to help chemists to better explore and understand interactions in chemical systems.

\section{Supplemental information}

IGM and IGMH maps of dodecaphenyltetracene at $\delta g^{\text {inter }}=0.003$ a.u.; NCI map of [12]CPP: $\mathrm{C}_{18}$ complex; Another perspective of Fig. 6; IGM map of 3b; IGMH map of COF with consideration of periodicity in all directions; IRI map of tetra(trimethylsilyl)tetrahedrane

\section{Funding information}

This research was not funded.

\section{Conflict of interest}

The authors declare no conflict of interest.

\section{References}

1. E. R. Johnson; S. Keinan; P. Mori-Sánchez; J. Contreras-García; A. J. Cohen; W. Yang. Revealing Noncovalent Interactions. J. Am. Chem. Soc., 132, 6498-6506 (2010). DOI: 10.1021/ja100936w.

2. T. Lu; S. Manzetti. Wavefunction and reactivity study of benzo[a]pyrene diol epoxide and its enantiomeric forms. Struct. Chem., 25, 1521-1533 (2014). DOI: 10.1007/s11224-014-0430-6.

3. S. Manzetti; T. Lu. The geometry and electronic structure of Aristolochic acid: possible implications for a frozen resonance. J. Phys. Org. Chem., 26, 473-483 (2013). DOI: 10.1002/poc.3111.

4. Z. Liu; T. Lu; Q. Chen. Intermolecular interaction characteristics of the all-carboatomic ring, cyclo[18]carbon: Focusing on molecular adsorption and stacking. Carbon, 171, 514-523 (2021). DOI: 10.1016/j.carbon.2020.09.048.

5. I. González-Veloso; J. A. Carrazana-García; D. Josa; J. Rodríguez-Otero; E. M. Cabaleiro-Lago. NCI analysis of the interaction cation $\cdots \pi$ in complexes with molecular bowls derived from fullerene. Comput. Theor. Chem., 1053, 123-129 (2015). DOI: 10.1016/j.comptc.2014.10.012.

6. J. Contreras-García; E. R. Johnson; S. Keinan; R. Chaudret; J.-P. Piquemal; D. N. Beratan; W. Yang. NCIPLOT: A Program for Plotting Noncovalent Interaction Regions. J. Chem. Theory Comput., 7, 625632 (2011). DOI: 10.1021/ct100641a. 
7. T. Lu; F. Chen. Multiwfn: A Multifunctional Wavefunction Analyzer. J. Comput. Chem., 33, 580592 (2012). DOI: 10.1002/jcc.22885.

8. T. Lu; Q. Chen. Interaction Region Indicator: A Simple Real Space Function Clearly Revealing Both Chemical Bonds and Weak Interactions. Chemistry-Methods, 1, 231-239 (2021). DOI: $10.1002 / \mathrm{cmtd} .202100007$.

9. R. F. W. Bader; T. S. Slee; D. Cremer; E. Kraka. Description of Conjugation and Hyperconjugation in Terms of Electron Distributions. J. Am. Chem. Soc., 105, 5061-5068 (1983). DOI: 10.1021/ja00353a035.

10. X. Mu; H. Zong; L. Zhu; M. Sun. External Electric Field-Dependent Photoinduced Charge Transfer in a Donor-Acceptor System in Two-Photon Absorption. J. Phys. Chem. C, 124, 2319-2332 (2020). DOI: 10.1021/acs.jpcc.9b10086.

11. J. Wang; J. Wang; X. Mu. Physical mechanism of concentration-dependent fluorescence resonance energy transfer. Spectrochim. Acta, Part A, 231, 118143 (2020). DOI: 10.1016/j.saa.2020.118143.

12. M. Wen; S. Li; J. Han; Y. Xue. Mechanism and enantioselectivity of the asymmetric [3+2]annulation between $\mathrm{N}$-methylindole and enoldiazoacetamide catalyzed by prolinate-coordinated dirhodium: A theoretical study. J. Mol. Graph. Model., 94, 107489 (2020). DOI: 10.1016/j.jmgm.2019.107489.

13. Q.-X. Zhang; Y. Li; J. Wang; C. Yang; C.-J. Liu; X. Li; J.-P. Cheng. B(C6F5)3/Chiral Phosphoric Acid Catalyzed Ketimine-Ene Reaction of 2-Aryl-3H-indol-3-ones and $\alpha$-Methylstyrenes. Angew. Chem. Int. Ed., 59, 4550-4556 (2020). DOI: 10.1002/anie.201915226.

14. Z. Zhang; L. He; J. Feng; X. Liu; L. Zhou; H. Zhang. Unveiling the Relationship between Energy Transfer and the Triplet Energy Level by Tuning Diarylethene within Europium(III) Complexes. Inorg. Chem., 59, 661-668 (2020). DOI: 10.1021/acs.inorgchem.9b02907.

15. W. Liu; S. Bobbala; C. L. Stern; J. E. Hornick; Y. Liu; A. E. Enciso; E. A. Scott; J. F. Stoddart. XCage: A Tricyclic Octacationic Receptor for Perylene Diimide with Picomolar Affinity in Water. J. Am. Chem. Soc., 142, 3165-3173 (2020). DOI: 10.1021/jacs.9b12982.

16. Z. Liang; H. Wang; X. Zheng; X. Wang. Theoretical investigation on rotaxanes containing a pyridylacyl hydrazone moiety: chemical $\mathrm{Z} \rightarrow \mathrm{E}$ and photochemical $\mathrm{E} \rightarrow \mathrm{Z}$ isomerizations. Theor. Chem. Acc., 139, 39 (2020). DOI: 10.1007/s00214-020-2553-8.

17. J. Li; A. C. Wachemo; G. Yu; X. Li. Enhanced anaerobic digestion performance of corn stalk pretreated with freezing-thawing and ammonia: An experimental and theoretical study. J. Clean. Prod., 247, 119112 (2020). DOI: 10.1016/j.jclepro.2019.119112.

18. H. Li; X. Zheng; Z. Jia; X. Wang. Theoretical study on noncovalent interaction of molecular tweezers by $\mathrm{Zn}(\mathrm{II})$ salphen-azo-crown ether triads receptor. J. Mol. Model., 26, 39 (2020). DOI: 10.1007/s00894-020-4301-3.

19. Q. Huang; K. Chai; L. Zhou; H. Ji. A phenyl-rich $\beta$-cyclodextrin porous crosslinked polymer for efficient removal of aromatic pollutants: Insight into adsorption performance and mechanism. Chem. Eng. J., 387, 124020 (2020). DOI: 10.1016/j.cej.2020.124020.

20. S. Geng; Z. Jiang; H. Ma; Y. Wang; B. Liu; G. Liang. Interaction mechanism of flavonoids and bovine $\beta$-lactoglobulin: Experimental and molecular modelling studies. Food Chem., 312, 126066 (2020). DOI: 10.1016/j.foodchem.2019.126066.

21. J. Zhang; Q. Bai; H. Pérez-Sánchez; S. Shang; X. An; X. Yao. Investigation of ECD conformational transition mechanism of GLP-1R by molecular dynamics simulations and Markov state model. Phys. Chem. Chem. Phys., 21, 8470-8481 (2019). DOI: 10.1039/C9CP00080A. 
22. K. Zhang; J. Liu; Y. Zhang; J. Fan; C.-K. Wang; L. Lin. Theoretical Study of the Mechanism of Aggregation-Caused Quenching in Near-Infrared Thermally Activated Delayed Fluorescence Molecules: Hydrogen-Bond Effect. J. Phys. Chem. C, 123, 24705-24713 (2019). DOI: 10.1021/acs.jpcc.9b06388.

23. P. R. Varadwaj; A. Varadwaj; H. M. Marques. C70 Fullerene Cage as a Novel Catalyst for Efficient Proton Transfer Reactions between Small Molecules: A Theoretical study. Sci. Rep., 9, 10650 (2019). DOI: $10.1038 / \mathrm{s} 41598-019-46725-4$.

24. I. K. Petrushenko; K. B. Petrushenko. Physical adsorption of hydrogen molecules on single-walled carbon nanotubes and carbon-boron-nitrogen heteronanotubes: A comparative DFT study. Vacuum, 167, 280-286 (2019). DOI: 10.1016/j.vacuum.2019.06.021.

25. H. Bian; A. Kan; Z. Yao; Z. Duan; H. Zhang; S. Zhang; L. Zhu; D. Xia. Impact of Functional Group Methylation on the Disaggregation Trend of Asphaltene: A Combined Experimental and Theoretical Study. J. Phys. Chem. C, 123, 29543-29555 (2019). DOI: 10.1021/acs.jpcc.9b07695.

26. T. Lu; Z. Liu; Q. Chen. Comment on "18 and 12 - Member carbon rings (cyclo[n]carbons) - A density functional study". Mat. Sci. Eng. B, 273, 115425 (2021). DOI: 10.1016/j.mseb.2021.115425.

27. C. Lefebvre; H. Khartabil; J.-C. Boisson; J. Contreras-García; J.-P. Piquemal; E. Hénon. The Independent Gradient Model: A New Approach for Probing Strong and Weak Interactions in Molecules from Wave Function Calculations. ChemPhysChem, 19, 724-735 (2018). DOI: 10.1002/cphc.201701325. 28. J. M. Flores-Álvarez; D. Cortés-Arriagada; J. Reyes-Gómez; Z. Gómez-Sandoval; J. C. Rojas-Montes; K. Pineda-Urbina. 2-Mercaptobenzothiazole modified carbon paste electrode as a novel copper sensor: An electrochemical and computational study. J. Electroanal. Chem., 888, 115208 (2021). DOI: 10.1016/j.jelechem.2021.115208.

29. C. Liu; H. Zhang; Y. Wang; H. Bai; D. Zhao; Q. Liu. Extraction separation of aromatic homologues from n-decane using DMSO: Influence of the alky side chain length. J. Chem. Thermodyn., 106673 (2021). DOI: 10.1016/j.jct.2021.106673.

30. C. Liu; D. Zhao; H. Zhang; Y. Wang; H. Bai; Q. Liu. Cumene extraction separation from alkanes using DMSO: Influence of the alkane structure. Fluid Phase Equilib., 550, 113236 (2021). DOI: 10.1016/j.fluid.2021.113236.

31. T. Zhu; P. Ning. Influence mechanism of supports for the adsorption and dissociation of $\mathrm{H} 2 \mathrm{O}$ and toluene in toluene steam reforming. Appl. Surf. Sci., 569, 151008 (2021). DOI: 10.1016/j.apsusc.2021.151008.

32. F. W. Bader. Atoms in Molecules: A Quantum Theory. Oxford University Press: New York, (1994).

33. C. Lefebvre; G. Rubez; H. Khartabil; J.-C. Boisson; J. Contreras-García; E. Hénon. Accurately extracting the signature of intermolecular interactions present in the NCI plot of the reduced density gradient versus electron density. Phys. Chem. Chem. Phys., 19, 17928-17936 (2017). DOI: 10.1039/C7CP02110K.

34. F. L. Hirshfeld. Bonded-Atom Fragments for Describing Molecular Charge Densities. Theor. Chem. Acc., 44, 129-138 (1977).

35. T. Lu; F. Chen. Atomic Dipole Moment Corrected Hirshfeld Population Method. J. Theor. Comp. Chem., 11, 163-183 (2012). DOI: 10.1142/S0219633612500113.

36. T. Lu; F. Chen. Comparison of Computational Methods for Atomic Charges. Acta Phys. -Chim. Sin.,

28, 1-18 (2012). DOI: 10.3866/PKU.WHXB2012281.

37. T. Lu; F. Chen. Bond Order Analysis Based on the Laplacian of Electron Density in Fuzzy Overlap Space. J. Phys. Chem. A, 117, 3100-3108 (2013). DOI: 10.1021/jp4010345.

38. M. Ponce-Vargas; C. Lefebvre; J.-C. Boisson; E. Hénon. Atomic Decomposition Scheme of 
Noncovalent Interactions Applied to Host-Guest Assemblies. J. Chem. Inf. Model., 60, 268-278 (2020). DOI: 10.1021/acs.jcim.9b01016.

39. R. M. Parrish; C. D. Sherrill. Spatial assignment of symmetry adapted perturbation theory interaction energy components: The atomic SAPT partition. J. Chem. Phys., 141, 044115 (2014). DOI: 10.1063/1.4889855.

40. R. M. Parrish; L. A. Burns; D. G. A. Smith; A. C. Simmonett; A. E. DePrince; E. G. Hohenstein; U. Bozkaya; A. Y. Sokolov; R. Di Remigio; R. M. Richard; J. F. Gonthier; A. M. James; H. R. McAlexander; A. Kumar; M. Saitow; X. Wang; B. P. Pritchard; P. Verma; H. F. Schaefer; K. Patkowski; R. A. King; E. F. Valeev; F. A. Evangelista; J. M. Turney; T. D. Crawford; C. D. Sherrill. Psi4 1.1: An Open-Source Electronic Structure Program Emphasizing Automation, Advanced Libraries, and Interoperability. $J$. Chem. Theory Comput., 13, 3185-3197 (2017). DOI: 10.1021/acs.jctc.7b00174.

41. W. Humphrey; A. Dalke; K. Schulten. VMD: Visual molecular dynamics. J. Mol. Graph., 14, 3338 (1996). DOI: 10.1016/0263-7855(96)00018-5.

42. A. D. Becke. A Multicenter Numerical Integration Scheme for Polyatomic Molecules. J. Chem. Phys., 88, 2547-2553 (1988). DOI: 10.1063/1.454033.

43. A. D. Becke; K. E. Edgecombe. A Simple Measure of Electron Localization in Atomic and Molecular Systems. J. Chem. Phys., 92, 5397-5403 (1990). DOI: 10.1063/1.458517.

44. T. Lu; F. Chen. Meaning and Functional Form of the Electron Localization Function. Acta Phys. Chim. Sin., 27, 2786-2792 (2011). DOI: 10.3866/PKU.WHXB20112786.

45. G. Jansen; B. A. Hess. Revision of the Douglas-Kroll Transformation. Phys. Rev. A, 39, 6016-6017 (1989).

46. T. Lu; Q. Chen. mwfn: A Strict, Concise and Extensible Format for Electronic Wavefunction Storage and Exchange. ChemRxiv (2020). DOI: 10.26434/chemrxiv.11872524.

47. T. D. Kühne; M. Iannuzzi; M. Del Ben; V. V. Rybkin; P. Seewald; F. Stein; T. Laino; R. Z. Khaliullin; O. Schütt; F. Schiffmann; D. Golze; J. Wilhelm; S. Chulkov; M. H. Bani-Hashemian; V. Weber; U. Borštnik; M. Taillefumier; A. S. Jakobovits; A. Lazzaro; H. Pabst; T. Müller; R. Schade; M. Guidon; S. Andermatt; N. Holmberg; G. K. Schenter; A. Hehn; A. Bussy; F. Belleflamme; G. Tabacchi; A. Glöß; M. Lass; I. Bethune; C. J. Mundy; C. Plessl; M. Watkins; J. VandeVondele; M. Krack; J. Hutter. CP2K: An electronic structure and molecular dynamics software package - Quickstep: Efficient and accurate electronic structure calculations. J. Chem. Phys., 152, 194103 (2020). DOI: 10.1063/5.0007045.

48. P. J. Stephens; F. J. Devlin; C. F. Chabalowski; M. J. Frisch. Ab Initio Calculation of Vibrational Absorption and Circular Dichroism Spectra Using Density Functional Force Fields. J. Phys. Chem., 98, 11623-11627 (1994). DOI: 10.1021/j100096a001.

49. S. Grimme; S. Ehrlich; L. Goerigk. Effect of the damping function in dispersion corrected density functional theory. J. Comput. Chem., 32, 1456-1465 (2011). DOI: 10.1002/jcc.21759.

50. J.-D. Chai; M. Head-Gordon. Long-range corrected hybrid density functionals with damped atomatom dispersion corrections. Phys. Chem. Chem. Phys., 10, 6615-6620 (2008). DOI: 10.1039/b810189b. 51. C. Bannwarth; S. Ehlert; S. Grimme. GFN2-xTB-An Accurate and Broadly Parametrized SelfConsistent Tight-Binding Quantum Chemical Method with Multipole Electrostatics and DensityDependent Dispersion Contributions. J. Chem. Theory Comput., 15, 1652-1671 (2019). DOI: 10.1021/acs.jctc.8b01176.

52. Stefan Grimme, et al., xtb program: https://github.com/grimme-lab/xtb/ (accessed on Nov 12, 2021).

53. M. J. Frisch; G. W. Trucks; H. B. Schlegel; G. E. Scuseria; M. A. Robb; J. R. Cheeseman; G. Scalmani; V. Barone; G. A. Petersson; H. Nakatsuji; X. Li; M. Caricato; A. V. Marenich; J. Bloino; B. G. 
Janesko; R. Gomperts; B. Mennucci; H. P. Hratchian; J. V. Ortiz; A. F. Izmaylov; J. L. Sonnenberg; Williams; F. Ding; F. Lipparini; F. Egidi; J. Goings; B. Peng; A. Petrone; T. Henderson; D. Ranasinghe; V. G. Zakrzewski; J. Gao; N. Rega; G. Zheng; W. Liang; M. Hada; M. Ehara; K. Toyota; R. Fukuda; J. Hasegawa; M. Ishida; T. Nakajima; Y. Honda; O. Kitao; H. Nakai; T. Vreven; K. Throssell; J. A. Montgomery Jr.; J. E. Peralta; F. Ogliaro; M. J. Bearpark; J. J. Heyd; E. N. Brothers; K. N. Kudin; V. N. Staroverov; T. A. Keith; R. Kobayashi; J. Normand; K. Raghavachari; A. P. Rendell; J. C. Burant; S. S. Iyengar; J. Tomasi; M. Cossi; J. M. Millam; M. Klene; C. Adamo; R. Cammi; J. W. Ochterski; R. L. Martin; K. Morokuma; O. Farkas; J. B. Foresman; D. J. Fox. Gaussian 16 A.03, Wallingford, CT, 2016. 54. R. Krishnan; J. S. Binkley; R. Seeger; J. A. Pople. Self-consistent molecular orbital methods. XX. A basis set for correlated wave functions. J. Chem. Phys., 72, 650-654 (1980).

55. J. P. Perdew; K. Burke; M. Ernzerhof. Generalized Gradient Approximation Made Simple. Phys. Rev. Lett., 77, 3865-3868 (1996).

56. Y. Xiao; J. T. Mague; R. H. Schmehl; F. M. Haque; R. A. Pascal Jr. Dodecaphenyltetracene. Angew. Chem. Int. Ed., 58, 2831-2833 (2019). DOI: 10.1002/anie.201812418.

57. S. Grimme. Do Special Noncovalent $\pi-\pi$ Stacking Interactions Really Exist? Angew. Chem. Int. Ed., 47, 3430-3434 (2008). DOI: 10.1002/anie.200705157.

58. K. Kaiser; L. M. Scriven; F. Schulz; P. Gawel; L. Gross; H. L. Anderson. An sp-hybridized molecular carbon allotrope, cyclo[18]carbon. Science, 365, 1299-1301 (2019).

59. Z. Liu; X. Wang; T. Lu; A. Yuan; X. Yan. Potential optical molecular switch: Lithium@cyclo[18]carbon complex transforming between two stable configurations. Carbon, 187, 7885 (2022). DOI: 10.1016/j.carbon.2021.11.005.

60. T. Lu; Z. Liu; Q. Chen. Comment on "18 and 12 - Member carbon rings (cyclo[n]carbons) - A density functional study”. Mat. Sci. Eng. B, 273, 115425 (2021). DOI: 10.1016/j.mseb.2021.115425.

61. T. Lu; Q. Chen. Ultrastrong Regulation Effect of the Electric Field on the All-Carboatomic Ring Cyclo[18]Carbon. ChemPhysChem, 22, 386-395 (2021). DOI: 10.1002/cphc.202000903.

62. Z. Liu; T. Lu; A. Yuan; X. Wang; Q. Chen; X. Yan. Remarkable Size Effect on Photophysical and Nonlinear Optical Properties of All-Carboatomic Rings, Cyclo[18]carbon and Its Analogues. Chem.Asian J., 16, 2267-2271 (2021). DOI: 10.1002/asia.202100589.

63. Z. Liu; T. Lu; Q. Chen. Vibrational spectra and molecular vibrational behaviors of all-carboatomic rings, cyclo[18]carbon and its analogues. Chem. - Asian J., 16, 56 (2021). DOI: 10.1002/asia.202001228. 64. Z. Liu; T. Lu; Q. Chen. Comment on "Theoretical investigation on bond and spectrum of cyclo[18]carbon (C18) with sp-hybridized”. J. Mol. Model., 27, 42 (2021). DOI: 10.1007/s00894-02104665-9.

65. Z. Liu; T. Lu; Q. Chen. An sp-hybridized all-carboatomic ring, cyclo[18]carbon: Bonding character, electron delocalization, and aromaticity. Carbon, 165, 468-475 (2020). DOI: 10.1016/j.carbon.2020.04.099.

66. Z. Liu; T. Lu; Q. Chen. An sp-hybridized all-carboatomic ring, cyclo[18]carbon: Electronic structure, electronic spectrum, and optical nonlinearity. Carbon, 165, $461-467$ (2020). DOI: 10.1016/j.carbon.2020.05.023.

67. Y. Jiang; E. J. Mattioli; M. Calvaresi; Z. Wang. Theoretical design of an ultrafast supramolecular rotor composed of carbon nano-rings. Chem. Commun., 56, 11835-11838 (2020). DOI: 10.1039/D0CC04806B.

68. S. Emamian; T. Lu; H. Kruse; H. Emamian. Exploring Nature and Predicting Strength of Hydrogen Bonds: A Correlation Analysis Between Atoms-in-Molecules Descriptors, Binding Energies, and Energy 
Components of Symmetry-Adapted Perturbation Theory. J. Comput. Chem., 40, 2868-2881 (2019). DOI: $10.1002 / \mathrm{jcc} .26068$.

69. G. Cavallo; P. Metrangolo; R. Milani; T. Pilati; A. Priimagi; G. Resnati; G. Terraneo. The Halogen Bond. Chem. Rev., 116, 2478-2601 (2016). DOI: 10.1021/acs.chemrev.5b00484.

70. F. Bulat; A. Toro-Labbé; T. Brinck; J. Murray; P. Politzer. Quantitative analysis of molecular surfaces: areas, volumes, electrostatic potentials and average local ionization energies. J. Mol. Model., 16, 1679-1691 (2010). DOI: 10.1007/s00894-010-0692-x.

71. L. P. Wolters; N. W. G. Smits; C. F. Guerra. Covalency in resonance-assisted halogen bonds demonstrated with cooperativity in N-halo-guanine quartets. Phys. Chem. Chem. Phys., 17, 1585-1592 (2015). DOI: 10.1039/C4CP03740E.

72. T. Hasell; X. Wu; J. T. A. Jones; J. Bacsa; A. Steiner; T. Mitra; A. Trewin; D. J. Adams; A. I. Cooper. Triply interlocked covalent organic cages. Nat. Chem., 2, 750-755 (2010). DOI: 10.1038/nchem.739.

73. S. Grimme; J. Antony; S. Ehrlich; H. Krieg. A consistent and accurate ab initio parametrization of density functional dispersion correction (DFT-D) for the 94 elements H-Pu. J. Chem. Phys., 132, 154104154119 (2010).

74. C. Adamo; V. Barone. Toward Reliable Density Functional Methods Without Adjustable Parameters: The PBE0 Model. J. Chem. Phys., 110, 6158-6170 (1999).

75. F. Weigend; R. Ahlrichs. Balanced basis sets of split valence, triple zeta valence and quadruple zeta valence quality for $\mathrm{H}$ to Rn: Design and assessment of accuracy. Phys. Chem. Chem. Phys., 7, 3297-3305 (2005). DOI: 10.1039/b508541a.

76. D. Cremer; E. Kraka. Chemical Bonds without Bonding Electron Density — Does the Difference Electron-Density Analysis Suffice for a Description of the Chemical Bond? Angew. Chem. Int. Ed. Engl., 23, 627-628 (1984). DOI: 10.1002/anie.198406271.

77. J. Yamane; M. Yao; Y. Zhou; Y. Hiramatsu; K. Fujiwara; T. Yamaguchi; H. Yamaguchi; H. Togame; H. Tsujishita; H. Takemoto; I. Tanaka. In-crystal affinity ranking of fragment hit compounds reveals a relationship with their inhibitory activities. J. Appl. Crystallogr., 44, 798-804 (2011). DOI: 10.1107/S0021889811017717.

78. J. D. Bernal; R. H. Fowler. A Theory of Water and Ionic Solution, with Particular Reference to Hydrogen and Hydroxyl Ions, 1, 515-548 (1933). DOI: 10.1063/1.1749327.

79. P. Politzer; J. S. Murray. The fundamental nature and role of the electrostatic potential in atoms and molecules. Theor. Chem. Acc., 108, 134-142 (2002). DOI: 10.1007/s00214-002-0363-9.

80. D. Ongari; A. V. Yakutovich; L. Talirz; B. Smit. Building a consistent and reproducible database for adsorption evaluation in Covalent-Organic Frameworks. Materials Cloud Archive, 2021.100 (2021). DOI: 10.24435/materialscloud:z6-jn.

81. T. Lu; Q. Chen. Revealing Molecular Electronic Structure via Analysis of Valence Electron Density. Acta Phys. -Chim. Sin., 34, 503-513 (2018). DOI: 10.3866/pku.Whxb201709252.

82. M. Giambiagi; M. de Giambiagi; K. Mundim. Definition of a multicenter bond index. Struct. Chem., 1, 423-427 (1990). DOI: 10.1007/bf00671228.

83. T. Lu; Q. Chen. A simple method of identifying $\pi$ orbitals for non-planar systems and a protocol of studying $\pi$ electronic structure. Theor. Chem. Acc., 139, 25 (2020). DOI: 10.1007/s00214-019-2541-z. 84. J. Pipek; P. G. Mezey. A fast intrinsic localization procedure applicable for ab initio and semiempirical linear combination of atomic orbital wave functions. J. Chem. Phys., 90, 4916-4926 (1989). DOI: 10.1063/1.456588. 